\title{
Hyperbolic Green Function Estimates
}

\author{
M. $\operatorname{Ryznar}^{1}$ (D). G. Serafin ${ }^{1} \cdot$ T. Żak ${ }^{1}$
}

Received: 10 July 2019 / Accepted: 4 February 2020 / Published online: 28 April 2020

(C) The Author(s) 2020

\begin{abstract}
For a hyperbolic Brownian motion in the hyperbolic space $\mathbb{H}^{n}, n \geq 3$, we prove a representation of a Green function and a Poisson kernel for bounded and smooth sets in terms of the corresponding objects for an ordinary Euclidean Brownian motion and a conditional gauge functional. Using this representation we prove bounds for the Green functions and Poisson kernels for smooth sets. In particular, we provide a two sided sharp estimate of the Green function of a hyperbolic ball of any radius. By usual isomorphism argument the same estimate holds in any other model of a real hyperbolic space.
\end{abstract}

Keywords Hitting times and distributions · Poisson kernels · Green functions · Hyperbolic spaces

Mathematics Subject Classification (2000) Primary 60J45; Secondary 60J60

\section{Introduction}

Potential analysis of a Brownian motion in $\mathbb{R}^{n}$ is developed and very well-known (compare for instance a book by Chung and Zhao [9]). In this classical context, for a sufficiently regular domain $D \subset \mathbb{R}^{n}$, there exist either formulas or very precise estimates of a Poisson kernel, a Green function and the distribution of the first exit/entrance time. From strictly analytical point of view the problem of behavior of the Green function of a set was considered in many papers. For instance in [15] it was proved that for a partial differential operator $L$ the ratio of $G_{L}^{D}$, the Green function of a bounded set $D$ with $C^{1,1}$ boundary and the Green function $G_{\Delta}^{D}$ of the same set for the ordinary Laplace operator $\Delta$ is bounded from below and from above by two positive numbers $C$ and $C^{-1}$, depending on the ellipticity constant of the operator $L$, the Hölder norm of coefficients of $L$, on the diameter of $D$ and the curvature of $\partial D$ :

$$
C^{-1} G_{\Delta}^{D}(x, y) \leq G_{L}^{D}(x, y) \leq C G_{\Delta}^{D}(x, y) .
$$

M. Ryznar

michal.ryznar@pwr.edu.pl

1 Faculty of Pure and Applied Mathematics, Wrocław University of Science and Technology, Wrocław, Poland 
The above estimate has one obvious disadvantage. As the size of $D$ grows, the constant $C$ usually blows up even for very regular sets like balls. Moreover, the inequality is not true if the domain is only Lipschitz (Remark 4.2 in [15]).

In the last fifteen years the similar problem was investigated for the generator of a hyperbolic Brownian motion. In several papers [4-8, 17, 20-23] various formulas or estimates were given not only for the Green function of a domain $D$ but also for the Poisson kernel of $D$ and for the distribution of the exit time of a process from $D$, provided $D$ is a hyperbolic ball or a half-space.

For instance, in the paper [23] the author used a spherical representation $(r, s)$ of a hyperbolic space, where $r$ is a distance from some fixed point $e$ and $s$ is a point on a hyperbolic sphere with center $e$ and radius 1. Applying an analytical method of separation of variables, he gave for $n \geq 3$ the following representation (with respect to the hyperbolic surface measure) of the Poisson kernel of a hyperbolic ball $B(e, R)$ of radius $R>0$ : for $x \in \partial B(e, r)$ and $z \in \partial B(e, R), 0<r<R$, let $\theta$ be an angle at $e$, formed by the geodesics connecting $e$ with $x$ and $e$ with $z$. Then

$$
P_{B(e, R)}(x, z)=P_{B(e, R)}(r, \theta)=\sum_{n=0}^{\infty} \frac{(n-2+2 k)}{(n-2)} \frac{\sinh ^{k}(r) F_{k}\left(-\sinh ^{2} \frac{r}{2}\right)}{\sinh ^{k}(R) F_{k}\left(-\sinh ^{2} \frac{R}{2}\right)} C_{k}^{\frac{n-2}{2}}(\cos \theta),
$$

where $C_{k}^{\frac{n-2}{2}}$ is an appropriate Gegenbauer polynomial and $F_{k}(t)={ }_{2} F_{1}\left(k, k+n-1, k+\frac{n}{2}, t\right)$ is a Gauss hypergeometric function. It seems to be impossible to use formula (2) to get precise estimate of $P_{B(e, R)}(r, \theta)$ in terms of simple elementary quantities as for example the distance $d_{\mathbb{H}^{n}}(x, z)$ and the distance of $x$ to the boundary of the ball.

Nevertheless, the formula (2) must be involved because, as it is proved in [23, Theorem $3]$, in dimension greater than 2, contrarily to the Euclidean case, in a hyperbolic space it is not possible to obtain the Poisson kernel $P_{B(e, R)}(x, z), x \in B(e, R), z \in \partial B(e, R)$, as a product of two functions of one variable: one of them being a function of the hyperbolic distance $d_{\mathbb{H}^{n}}(e, x)$ only and the second function depending only on the distance $d_{\mathbb{H}^{n}}(x, z)$.

The authors of [8] also computed the Poisson kernel of a ball, using only analytical methods of separation of variables and the spherical representation of a hyperbolic space. Their result is formulated in the language of the hyperbolic Brownian motion and the formula for the Poisson kernel is equivalent to that from [23], up to some obvious transformations.

We remark that in most of the above-listed papers probabilistic methods were used. Namely, a hyperbolic Brownian motion was described as a solution of a system of stochastic differential equations either in $\mathbb{H}^{n}=\left\{x \in \mathbb{R}^{n}:\left(x_{1}, . ., x_{n-1}\right) \in \mathbb{R}^{n-1}, x_{n}>0\right\}$, a half-space model or in $\mathbb{D}^{n}=\left\{x \in \mathbb{R}^{n} \quad|x|<1\right\}$, the unit ball model of a hyperbolic space. Using this approach one can get formulas for Poisson kernels and Green functions for some very regular domains. In particular in [5], the authors using the ball model of a hyperbolic space and stochastic calculus obtained not only the above mentioned formula for the Poisson kernel of a ball but also a formula for the Green function of a ball again in terms of a complicated series involving hypergeometric functions and Gegenbauer polynomials. However, it is not easy, if not impossible, to use these formulas to get precise estimates of the Poisson kernel or the Green function of a ball (compare computations for dimension $n=4$ and $n=6$ in Corollary 5.2 in [5]).

In the $\mathbb{H}^{n}$ model of the hyperbolic space the following representation of a hyperbolic Brownian motion $\mathbf{X}(t)=\left(X_{1}(t), \ldots, X_{n}(t)\right)$ in $\mathbb{H}^{n}$ was applied: the last coordinate $X_{n}(t)$ is an exponential of a linear Brownian motion with some drift (depending on the dimension 
of the space) and $\left(X_{1}(t), \ldots, X_{n-1}(t)\right)$ is an Euclidean Brownian motion in $\mathbb{R}^{n-1}$, timechanged by a random clock dictated by the last coordinate.

In this paper we propose a little different approach. We change a part of the drift of a hyperbolic Brownian motion by killing trajectories with some intensity and next we recover a process (rather than a sub-process), using Doob's $h$-transform with a function $h$ that is invariant for the killed process. In this way we find it possible to represent the Poisson kernel of any bounded (in the hyperbolic metric) domain $D$ with $C^{1,1}$ boundary in terms of the classical Poisson kernel of the domain. An analogous representation is obtained for Green functions. Namely, in Corollary 4.2, we describe the Poisson kernel of any such domain $D$ in $\mathbb{H}^{n}$ by the following formula:

$$
P_{D}(x, z)=\frac{h(z)}{h(x)} P_{D}^{\Delta}(x, z) n_{D}(x, z), x \in D, z \in \partial D,
$$

where $h(x)=x_{n}^{1-\frac{n}{2}}, P_{D}^{\Delta}(x, z)$ is the Poisson kernel of $D$, corresponding to the ordinary Laplacian $\Delta$, and

$$
n_{D}(x, y)=\mathbb{E}_{y}^{x} \exp \left(-\int_{0}^{\tau_{D}} \frac{n(n-2)}{4 W_{n}^{2}(t)} d t\right), x \in D, y \in \bar{D}
$$

is a conditional gauge functional, and $\mathbb{E}_{y}^{x}$ is some conditional expectation (see Section 4). Note that $n_{D}$ is bounded from above by 1 and possible to estimate from below for a large class of domains $D$. We also give an analogous description of the Green function of a bounded set $D$ with $C^{1,1}$ boundary:

$$
G_{D}(x, y)=\frac{1}{h(x) h(y)} n_{D}(x, y) G_{D}^{\Delta}(x, y), x \in D, y \in D .
$$

Using the above representation of the Green function of $D$, estimating the conditional gauge $n_{D}(x, y)$ by applying the results of [11], we obtain estimates similar to (1) (compare our Corollary 5.2), which might be considered as improvements of (1), especially the upper bound. As a consequence, in Theorem 5.7, we obtained a general upper bound for the Green function of a $C^{1,1}$ domain $D$ possibly not bounded.

Finally, in Theorem 6.1, which may be considered as the main result of the paper, we provide a precise two sided estimate of the Green function of a hyperbolic ball $B$ of any radius. While for small size balls one can take advantage of our representation or (1), then the case of large balls requires a substantial additional effort. Let $U(x, y), x, y \in \mathbb{H}^{n}$ be the potential kernel (with respect to the Riemannian volume measure in $\mathbb{H}^{n}$ ) of a hyperbolic Brownian motion, then we show in our Lemma 6.3 that $G_{B}(x, y) \geq C U(x, y)$, provided the distances of $x$ and $y$ from the boundary of $B$ are greater than some constant $C^{*}$. Here both $C$ and $C^{*}$ depend only on $n$.

In consequence, for a ball $B=B\left(x_{0}, R\right)$ we prove that for all $x, y \in B$,

$$
C^{-1}\left(1 \wedge \frac{\left(1 \wedge \delta_{B}(x)\right)\left(1 \wedge \delta_{B}(y)\right)}{1 \wedge d_{\mathbb{H}^{n}}(x, y)^{2}}\right) \leq \frac{G_{B}(x, y)}{U(x, y)} \leq C\left(1 \wedge \frac{\left(1 \wedge \delta_{B}(x)\right)\left(1 \wedge \delta_{B}(y)\right)}{1 \wedge d_{\mathbb{H}^{n}}(x, y)^{2}}\right),
$$

where the constant $C$ is dependent only on $n, \delta_{B}(x)$ denotes the hyperbolic distance from $x$ to $\partial B$ and $d_{\mathbb{H}^{n}}$ is the hyperbolic metric and, as was shown in [4],

$$
U(x, y) \approx \frac{1}{\left(\cosh d_{\mathbb{H}^{n}}(x, y)-1\right)^{\frac{n}{2}-1}\left(\cosh d_{\mathbb{H}^{n}}(x, y)\right)^{\frac{n}{2}}}, x, y \in \mathbb{H}^{n} .
$$


Throughout the whole paper, for two non-negative functions, $f(z) \approx g(z), z \in Z$, denotes that there exists a positive $C$, called a comparability constant, such that for all $z \in Z$,

$$
C^{-1} g(z) \leq f(z) \leq C g(z) .
$$

A comparison like (3) was obtained in [4] but only for half-spaces $D_{a}=\left\{x \in \mathbb{H}^{n}: x_{n}>\right.$ $a\}, a>0$. The methods of [4] are completely different than ours, since they rely on the estimates of the Bessel heat kernel for a half-line. On the other hand their result follows from ours since we can approach any half-space $D_{a}$ by a sequence of balls.

\section{Two Models of a Hyperbolic Space}

Let us define $\mathbb{H}^{n}=\left\{x \in \mathbb{R}^{n}: x_{n}>0\right\}$. This half-space of $\mathbb{R}^{n}$, equipped with the Riemannian metric $d s^{2}=\frac{d x_{1}^{2}+\ldots+d x_{n}^{2}}{x_{n}^{2}}$, is a model of $n$-dimensional hyperbolic space (see e.g. [18]). The volume element (the hyperbolic reference measure) is given by $d \mu=\frac{d x_{1} \ldots d x_{n}}{x_{n}^{n}}$ and the hyperbolic distance $d_{\mathbb{H}^{n}}(x, y)$ is given by

$$
\cosh \left(d_{\mathbb{H}^{n}}(x, y)\right)=1+\frac{|x-y|^{2}}{2 x_{n} y_{n}},
$$

where $|x-y|$ denotes the Euclidean distance. Since $\mathbb{H}^{n}$ is a subset of $\mathbb{R}^{n}$, sometimes we will consider this set equipped with the Euclidean metric. In order to simplify notation and avoid misunderstanding, for $x, y \in \mathbb{H}^{n}$ we will denote by $|x-y|$ their Euclidean distance and by $d_{\mathbb{H}^{n}}(x, y)$ their hyperbolic distance.

From the formula (4) it is easy to see that a hyperbolic ball in $\mathbb{H}^{n}$ is also a Euclidean ball but with different center and radius. In particular, a hyperbolic ball $B((0, . ., 0,1), R)$, $R>0$, is an Euclidean ball with center $(0, \ldots, 0, \cosh R)$ and radius $\sinh R$.

For one-dimensional space $\mathbb{H}^{1}$ formula (4) gives the following: for $x, y \in \mathbb{H}^{1}$ (that is for $x, y \in(0, \infty))$

$$
d_{\mathbb{H}^{1}}(x, y)=\left|\log \frac{x}{y}\right|,
$$

hence an open ball $B(1, R) \subset \mathbb{H}^{1}$ is an interval $\left(e^{-R}, e^{R}\right)$. Moreover, for points $x, y \in \mathbb{R}^{k}$, $k \leq n$, their distance in $\mathbb{H}^{k}$ given by Eq. 4 is equal to the distance between $\left(0, \ldots, x_{1}, \ldots, x_{k}\right)$ and $\left(0, \ldots, 0, y_{1}, \ldots, y_{k}\right)$ as points from $\mathbb{H}^{n}$.

The group of isometries of $\mathbb{H}^{n}$ is generated by Euclidean translations parallel to the Euclidean boundary of $\mathbb{H}^{n}$, symmetries with respect to hyperplanes perpendicular to the boundary of $\mathbb{H}^{n}$ and by inversions of $\mathbb{H}^{n}$ with respect to half-spheres with centers on the boundary of $\mathbb{H}^{n}$ (see e.g. [18]). In particular, for any two points $A, B$ on a hyperbolic sphere $S\left(x_{0}, R\right)=\left\{x \in \mathbb{H}^{n}: d_{\mathbb{H}^{n}}\left(x, x_{0}\right)=R\right\}$ there exists a hyperbolic isometry $I_{A B}$ such that $I_{A B}\left(S\left(x_{0}, R\right)\right)=S\left(x_{0}, R\right)$ and $I_{A B}(A)=B$. Next we may isometrically translate $S\left(x_{0}, R\right)$ such that its center $x_{0}$ has new coordinates $\left(0, \ldots, 0,\left(x_{0}\right)_{n}\right)$. Then, using an inversion with respect to a sphere $S\left(0, \frac{1}{\sqrt{\left(x_{0}\right)_{n}}}\right)$, we transform the ball with center $\left(0, \ldots, 0,\left(x_{0}\right)_{n}\right)$ and radius $R$ onto a ball with center $(0, \ldots, 0,1)$ and radius $R$.

The above-mentioned rotations are in general given by complicated formulas. In a very

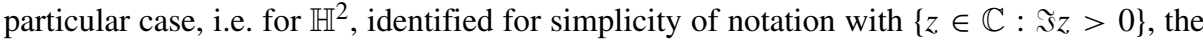
hyperbolic rotation by angle $\theta$ around $i$ is given by the following simple formula

$$
I_{\theta}(z)=\frac{\cos \frac{\theta}{2} z+\sin \frac{\theta}{2}}{-\sin \frac{\theta}{2} z+\cos \frac{\theta}{2}} .
$$


Combining hyperbolic isometries described above, we get the following fact, crucial for our future considerations:

Proposition 2.1 Any hyperbolic ball $B\left(x_{0}, R\right) \subset \mathbb{H}^{n}$ can be isometrically transformed onto a ball with center $(0, \ldots, 0,1)$ and radius $R$. Moreover, if $y$ is any fixed point of a sphere $S\left(x_{0}, R\right)$ then there exists a hyperbolic isometry I such that $I\left(x_{0}\right)=(0, \ldots, 0,1)$ and $I(y)=\left(0, \ldots, 0, e^{-R}\right)$.

Furthermore, it is possible to find such isometry of $\mathbb{H}^{n}$ that transforms a ball $B((0, \ldots, 0,1), R)$ onto the same ball but in such a way that any two distinct points from the ball after this transformation have the same last coordinate.

Proposition 2.2 Let $B$ be a ball in $\mathbb{H}^{n}$ with the radius $R$ and the center $(0, \ldots, 0,1)$. Let $x, y$ be two distinct points of $B$. Then there exists an isometry $I$ of $\mathbb{H}^{n}$ such that $I(B)=B$ and the last coordinate of $I(x)$ is equal to the last coordinate of $I(y)$.

Before we give a proof of the above proposition, we recall the unit ball model of $n$ dimensional hyperbolic space (see [18]), because in this model the proof is simpler. Let $\mathbb{D}^{n}=\left\{x \in \mathbb{R}^{n}:|x|<1\right\}$ be the unit ball of $\mathbb{R}^{n}$ equipped with a metric $d s^{2}=\frac{d x_{1}^{2}+\ldots+d x_{n}^{2}}{\left(1-|x|^{2}\right)^{2}}$. The set of isometries of $\mathbb{D}^{n}$ consists of all rotations around the origin (i.e. all orthogonal transformations), symmetries with respect to the hyperplanes containing the origin and inversions with respect to all spheres orthogonal to $S(0,1)$, the unit sphere in $\mathbb{R}^{n}$, that is the Euclidean boundary of $\mathbb{D}^{n}$. A horosphere in $\mathbb{D}^{n}$ is a sphere, contained in $\mathbb{D}^{n}$ and tangent to $S(0,1)$. The inversion of $\mathbb{R}^{n} \backslash\{(0, \ldots, 0,-1)\}$ with respect to a sphere with Euclidean radius $\sqrt{2}$ and center $(0, \ldots, 0,-1)$ is an isometry of Riemannian structures of $\mathbb{H}^{n}$ and $\mathbb{D}^{n}$. This inversion transports all horospheres in $\mathbb{D}^{n}$ that are tangent to $S(0,1)$ at its "south pole", i.e. at the point $(0, \ldots, 0,-1)$, onto a set of all Euclidean hyperplanes parallel to the Euclidean boundary of $\mathbb{H}^{n}$ (see [18]).

Proof of Proposition 2.2 We use the above described isometry of both models of a hyperbolic space. First, observe that the inversion transforming $\mathbb{H}^{n}$ onto $\mathbb{D}^{n}$ transforms the ball $B$ onto a ball, say $K$, with center $(0, \ldots, 0)$ and hyperbolic radius $R$ in $\mathbb{D}^{n}$. Let $x$ and $y$ be two distinct points in $K$. We want to prove that there exists a horosphere $S$ in $\mathbb{D}^{n}$ such that $x$ and $y$ are on the horosphere. Points $x, y$ and the origin determine a two-dimensional plane in $\mathbb{R}^{n}$. Using an appropriate rotation if needed, we can assume that all points on this two-dimensional plane are of the following form $\left(0, \ldots, 0, x_{n-1}, x_{n}\right)$. In this way we have to solve the following two-dimensional problem: having the unit circle on the Euclidean plane and two distinct points inside the circle, find a circle passing through $x$ and $y$ and tangent to the unit circle. We consider two cases:

1. $|x|=|y|$,

2. $|x|>|y|$.

If $|x|=|y|$ then there exists an isosceles triangle with vertices $x, y$ and having the third vertex $s^{*}$ on the unit circle. Then a circle circumscribed on this triangle is a horocycle we were looking for.

If $|x|>|y|$ then let us draw a line passing through $x$ and the origin and let $s_{1}, s_{2}$ be the points where the line intersects the unit circle. Assume that $\left|x-s_{1}\right|>\left|x-s_{2}\right|$. Because $|x|>|y|$, a circle such that the interval $\overline{x s_{1}}$ is its diameter encircle point $y$ and the circle with diameter $\overline{x s_{2}}$ does not encircle $y$. For points $s \in S(0,1)$ let us consider a family of all 
circles inscribed in $S(0,1)$, tangent to $S(0,1)$ at $s$ and passing through $x$. Because for $s_{1}$ the circle encircles $y$ and for $s_{2}$ it does not, continuity implies that there exists $s^{*} \in S(0,1)$ such that the circle from the family, determined by $s^{*}$ passes through $y$. Having such horosphere (or horocycle), we rotate $\mathbb{D}^{n}$ in such a way that $s^{*}$ is transformed on the "south pole" of the unit sphere. The isometry between $\mathbb{D}^{n}$ and $\mathbb{H}^{n}$, described above, transports such horosphere onto a hyperplane parallel to the Euclidean boundary of $\mathbb{H}^{n}$. This means that after these transformations, all of which are isometries of a hyperbolic space, the last coordinates of $x$ and $y$ are equal.

\section{Hyperbolic Brownian Motion}

The Laplace-Beltrami operator in $\mathbb{H}^{n}$ is given by the following formula (compare [14] or [18])

$$
\Delta_{\mathbb{H}^{n}}=x_{n}^{2}\left(\sum_{k=1}^{n} \frac{\partial^{2}}{\partial x_{k}^{2}}\right)-(n-2) x_{n} \frac{\partial}{\partial x_{n}} .
$$

This operator commutes with all isometries of $\mathbb{H}^{n}:$ if $I$ is such isometry, then for all $f \in C^{2}\left(\mathbb{H}^{n}\right)$ there holds

$$
\Delta_{\mathbb{H}^{n}}(f \circ I)=\left(\Delta_{\mathbb{H}^{n}} f\right) \circ I .
$$

A hyperbolic Brownian motion $\mathbf{B}(t)$ in $\mathbb{H}^{n}$ is a strong Markov process generated by $\Delta_{\mathbb{H}^{n}}$. Because $\Delta_{\mathbb{H}^{n}}$ commutes with isometries of $\mathbb{H}^{n}$, the hyperbolic Brownian motion $\mathbf{B}(t)$ starting from $x \in \mathbb{H}^{n}$ is invariant with respect to all hyperbolic isometries, leaving point $x$ invariant (similarly, as the classical Brownian motion starting from $x \in \mathbb{R}^{n}$ is invariant with respect to all Euclidean isometries of $\mathbb{R}^{n}$, leaving point $x$ invariant). To avoid misunderstanding we will denote an Euclidean Brownian motion generated by the Laplacian $\Delta$ (with values in $\mathbb{R}$ or in $\mathbb{R}^{n}$ ) by $W(t)$.

The hyperbolic Brownian motion in $\mathbb{H}^{n}$ has a transition density (with respect to the Riemannian measure $d \mu$ ) that depends on the hyperbolic distance between $x$ and $y: p_{t}(x, y)=$ $p_{t}\left(d_{\mathbb{H}^{n}}(x, y)\right)$. For odd dimensions this density is an elementary function, for even dimensions it is not elementary but its precise estimates are well-known [12]. The potential of the process (with respect to $d \mu$ ) is finite for all $n \geq 2$ and its kernel depends only on $d_{\mathbb{H}^{n}}(x, y)$ : $U(x, y)=\int_{0}^{\infty} p_{t}\left(d_{\mathbb{H}^{n}}(x, y)\right) d t<\infty$.

We are interested in processes killed on exiting a set $D$. Let $X(t)$ be a stochastic process and let $D$ be an open set in $\mathbb{H}^{n}$. We define the first exit time of $X$ from $D$ as

$$
\tau_{D}=\inf \{t: X(t) \notin D\},
$$

and the process killed on exiting $D$ by

$$
X^{D}(t)= \begin{cases}X(t), & t<\tau_{D}, \\ \delta, & t \geqslant \tau_{D},\end{cases}
$$

where $\delta$ is a 'cemetery point', i.e. some isolated point. If $X(t)$ is Markov and has a transition density $p(t ; x, y)$, then the killed process also has a transition density, given by Hunt's formula [16]:

$$
p^{D}(t ; x, y)=p(t ; x, y)-\mathbb{E}^{x}\left[\tau_{D}<t ; p\left(t-\tau_{D} ; X\left(\tau_{D}\right), y\right)\right],
$$

where $\mathbb{E}^{x}$ is an expectation with respect to the distribution $\mathbb{P}^{x}$ of the process starting from $x$. We call $p^{D}(t ; x, y)$ the Dirichlet heat kernel of $X$ on $D$. 
We will also use $G_{D}(x, y)$, the Green function of $X$ on $D$. It is given by the following formula

$$
G_{D}(x, y)=\int_{0}^{\infty} p^{D}(t ; x, y) d t,
$$

whenever the integral is convergent.

One can also kill trajectories of a process $X$ according to an additive functional with intensity $q(x)$, where the function $q(x)$ is usually called a 'potential' (compare [1]). Then the killed process is still a Markov process and its generator is given by $L^{q} f(x)=L f(x)-$ $q(x) f(x)$, where $L$ is the generator of the original process $X$. Equation $L^{q} u(x)=L u(x)-$ $q(x) u(x)=0$ is called the Schrödinger equation and for functions $q(x)$ belonging to a Kato class the appropriate Feynman-Kac semigroup $\left(T_{t}\right)$ of operators is given by the following formula (see e.g. [9], chapter 3):

$$
T_{t} u(x)=\mathbb{E}^{x}\left[e^{-\int_{0}^{t} q\left(X_{s}\right) d s} u\left(X_{t}\right)\right] .
$$

Moreover, combining both types of killing trajectories of a process $X$, from the semigroup $\left(P_{t}\right)$ of operators for process $X$ we first get the semigroup $\left(P_{t}^{D}\right)$ of operators for the process killed on exiting $D$, and next the Feynman-Kac semigroup $\left(T_{t}^{D}\right)$ for the process $\left(X_{t}^{D}\right)$, whose trajectories are killed with some potential $q(x)$ and on exiting $D$ (compare [9]):

$$
T_{t}^{D} u(x)=\mathbb{E}^{x}\left[e^{-\int_{0}^{t} q\left(X_{s}^{D}\right) d s} u\left(X_{t}^{D}\right)\right]=\mathbb{E}^{x}\left[e^{-\int_{0}^{t} q\left(X_{s}\right) d s} u\left(X_{t}\right) ; t<\tau_{D}\right] .
$$

We will also use the Doob $h$-transform, which is defined for an excessive function $h$. Recall that a Borel function $h$ is called excessive for a process $X$ if $\mathbb{E}_{x} h\left(X_{t}\right) \leq h(x)$ for all $x$ and $t$ and $\lim _{t \rightarrow 0+} \mathbb{E}_{x} h\left(X_{t}\right)=h(x)$ for all $x$. An excessive function is said to be invariant if $\mathbb{E}_{x} h\left(X_{t}\right)=h(x)$ for all $x$ and $t$. Let $D \subset \mathbb{H}^{n}$ be an open set. A Borel function $h$ on $\mathbb{H}^{n}$ is called excessive (invariant) on $D$ if it is excessive (invariant) for the process $X$ killed when it exits $D$.

Let $h$ be an excessive function and set $E_{h}=\left\{x \in \mathbb{H}^{n}: 0<h(x)<\infty\right\}$. Following [10], we can define the Doob $h$-transform $\left(X_{t}^{h}\right)$ of $\left(X_{t}\right)$ as the Markov or sub-Markovian process with transition semigroup prescribed by

$$
P_{t}^{h}(x, d y)= \begin{cases}\frac{h(y)}{h(x)} P_{t}^{K}(x, d y) & \text { if } \quad x \in E_{h} ; \\ 0 & \text { if } \quad x \in \mathbb{H}^{n} \backslash E_{h},\end{cases}
$$

where $\left(P_{t}^{K}(x, d y)\right)$ is the semigroup of $X$ killed upon exiting $E_{h}$.

We will use a harmonic function $h$ that is finite and strictly positive everywhere on $\mathbb{H}^{n}$ so that we will always have $P_{t}^{h}(x, d y)=\frac{h(y)}{h(x)} P_{t}(x, d y)$.

\section{A Representation of a Hyperbolic Brownian Motion in $\mathbb{H}^{n}$}

A hyperbolic Brownian motion in $\mathbb{H}^{n}$ is a diffusion $\mathbf{B}(t)=\left(\mathbf{B}_{1}(t), \ldots, \mathbf{B}_{n}(t)\right)$ with the generator $\Delta_{\mathbb{H}^{n}}$. Observe that in dimension one $\mathbf{B}(t)$ is simply an exponent of one-dimensional Brownian motion: $\mathbf{B}(t)=e^{W(t)}$, where $\mathbb{E}^{0}\left(W^{2}(t)\right)=2 t$. This implies that every question concerning potential theory of Brownian motion in $\mathbb{H}^{1}$ can be easily translated to Euclidean one-dimensional Brownian context and answered there.

If $n=2$, we have $\Delta_{\mathbb{H}^{n}}=x_{2}^{2} \Delta$, so that two-dimensional hyperbolic Brownian motion is an Euclidean planar Brownian motion $\left(W_{1}, W_{2}\right)$, killed on exiting $\mathbb{H}^{2}=\left\{x \in \mathbb{R}^{2}: x_{2}>0\right\}$ and time-changed by a functional $A(t)=\int_{0}^{t} e^{2\left(W_{2}(s)-s\right)} d s$. This implies that questions 
concerning notions from potential theory such as description of a Poisson kernel or a Green function of a regular domain on the plane can be, as in dimension one, easily translated to the Euclidean context, where the answers are usually known. Thus we will assume below that $n \geq 3$.

Using the well-known connections between generators of diffusions and systems of stochastic differential equations, we can define $\mathbf{B}(t)$ as a strong solution of the following system:

$$
\begin{cases}d \mathbf{B}_{1}(t) & =\mathbf{B}_{n}(t) d W_{1}(t), \\ d \mathbf{B}_{2}(t) & =\mathbf{B}_{n}(t) d W_{2}(t), \\ \cdots & \cdots \\ d \mathbf{B}_{n-1}(t) & =\mathbf{B}_{n}(t) d W_{n-1}(t), \\ d \mathbf{B}_{n}(t) & =\mathbf{B}_{n}(t) d W_{n}(t)-(n-2) \mathbf{B}_{n}(t) d t,\end{cases}
$$

where $\left(W_{1}, \ldots, W_{n}\right)$ is an Euclidean Brownian motion with $\operatorname{Var}\left(W_{k}(t)\right)=2 t, k=1, \ldots, n$.

Using Itô's formula we see that the solution of the last equation is an exponential Brownian motion with drift: $\mathbf{B}_{n}(t)=\exp \left(W_{n}(t)-(n-1) t\right)$. Solutions of equations describing $\mathbf{B}_{1}, \ldots, \mathbf{B}_{n-1}$ can be written in the following form: if $W(t)=\left(W_{1}(t), \ldots, W_{n-1}(t)\right)$ denotes an Euclidean Brownian motion in $\mathbb{R}^{n-1}$ then $\left(\mathbf{B}_{1}(t), \ldots, \mathbf{B}_{n-1}(t)\right)=W\left(A_{n}(t)\right)$, where the time change is given by the functional

$$
A_{n}(t)=\int_{0}^{t} \exp \left(2\left(W_{n}(s)-(n-1) s\right)\right) d s,
$$

(compare [7] or [4] for more details on this representation and [13] for a random timechange of a process).

Summing up: the above representation describes a hyperbolic Brownian motion in $\mathbb{H}^{n}$ as a time-changed Euclidean Brownian motion in $\mathbb{R}^{n-1}$ with the $n$-th coordinate being geometric Brownian motion $\mathbf{B}_{n}(t)=\exp \left(W_{n}(t)-(n-1) t\right)$, where the new clock $A_{n}(t)$ is dictated by the $n$-th coordinate. Observe that the drift term in the last coordinate depends on $n$ and is equal to $(n-1) t$. This representation helped the authors of [4] give sharp estimates of the $\lambda$-Green function of a set $\left\{\left(x_{1}, \ldots, x_{n}\right): x_{n}>a\right\}, a>0$.

We propose here a little different description of a hyperbolic Brownian motion in $\mathbb{H}^{n}$, $n \geq 3$. Consider an operator $L_{1}$, given for twice differentiable $f$ with compact support in $\mathbb{H}^{n}$ by the following formula:

$$
L_{1} f(x)=x_{n}^{2} \Delta f(x), x \in \mathbb{H}^{n},
$$

where $\Delta$ is the Laplace operator in $\mathbb{R}^{n}$. Operator $\Delta$ is the generator of a Brownian motion $\left(W_{1}(t), \ldots, W_{n}(t)\right)$ in $\mathbb{R}^{n}$ but multiplied by $x_{n}^{2}$ is the generator of a diffusion $\mathbf{X}(t)=$ $\left(X_{1}(t), \ldots, X_{n}(t)\right)$, living in $\mathbb{R}^{n-1} \times(0, \infty)$ (if started in this half-space).

Let us note that the process $\mathbf{X}(t)$ is a solution of system analogous to Eq. 6 with the last equation replaced by $d X_{n}(t)=X_{n}(t) d W_{n}(t)$. Observe also that the solution of this equation is given by $X_{n}(t)=e^{W_{n}(t)-t}$.

Recall that if $Y(t)$ is a process with generator $\mathcal{G}$ and $\tau(t)=\int_{0}^{t} \xi(Y(s)) d s$, where $\xi$ is a positive, continuous function, then the process $Y(\tau(t))$ is also a Markov process with generator $\xi \mathcal{G}$ (compare [13]). In our case $\xi\left(x_{1}, \ldots, x_{n}\right)=x_{n}^{2}$ and $X_{n}(t)=e^{W_{n}(t)-t}$ so that $\tau(t)$ is equal to

$$
\tau(t)=\int_{0}^{t} e^{2\left(W_{n}(s)-s\right)} d s=A_{2}(t) .
$$

Hence the processes $\left(X_{1}(t), \ldots, X_{n-1}(t), X_{n}(t)\right),\left(W_{1}\left(A_{2}(t)\right), \ldots, W_{n-1}\left(A_{2}(t)\right), W_{n}\left(A_{2}(t)\right)\right)$ and $\left(W_{1}\left(A_{2}(t)\right), \ldots, W_{n-1}\left(A_{2}(t)\right), e^{W_{n}(t)-t}\right)$ have the same distributions. 
Let us kill trajectories of the process $\mathbf{X}(t)$ with constant intensity $q_{0}(x)=\frac{n(n-2)}{4}$. This means that for $f(x) \in C^{2}\left(\mathbb{H}^{n}\right)$ and with compact support the generator of the killed process, call it $L_{2}$, is equal to

$$
L_{2} f(x)=x_{n}^{2} \Delta f(x)-\frac{n(n-2)}{4} f(x)=x_{n}^{2}\left(\Delta-q_{1}\right) f(x),
$$

where $q_{1}(x)=q_{1}\left(x_{n}\right)=\frac{n(n-2)}{4 x_{n}^{2}}, x \in \mathbb{H}^{n}$. This is the generator of a subprocess $\mathbf{X}^{*}(t)$ living in $\mathbb{H}^{n}$, which means that $\mathbb{P}\left(\mathbf{X}^{*}(t) \in \mathbb{H}^{n}\right)<1$ for all $t>0$. Using Doob's $h$-transform we want to condition the killed process in such a way, that its last coordinate will tend to zero, when time passes to infinity. This means that we are looking for a function $h(x)=h\left(x_{n}\right)$, depending only on the last coordinate, with a pole at zero and such that $L_{2} h(x)=0$. Solving the equation

$$
h^{\prime \prime}\left(x_{n}\right)-\frac{n(n-2)}{4 x_{n}^{2}} h\left(x_{n}\right)=0,
$$

we get $h\left(x_{n}\right)=c_{1} x_{n}^{n / 2}+c_{2} x_{n}^{1-\frac{n}{2}}$. We choose $c_{1}=0$ and $c_{2}=1$ and get the desired harmonic function: $h\left(x_{n}\right)=x_{n}^{1-\frac{n}{2}}$. Because for $x_{n} \in(0, \infty)$ the function $h$ is finite and strictly positive, hence in Eq. 5 no additional killing is needed.

Function $h$ is not only harmonic but it is also invariant for a process $X_{n}^{*}(t)$ with generator $x_{n}^{2} \frac{d^{2}}{d x_{n}^{2}}-\frac{n(n-2)}{4}$, hence also for the operator $L_{2}$. Indeed, let $\left(T_{t}^{*}\right)_{t \geq 0}$ be the Feynman-Kac semigroup for the process $e^{W(t)-t}$ killed with intensity $q_{0}\left(x_{n}\right)=\frac{n(n-2)}{4}$. Then

$$
T_{t}^{*} f\left(x_{n}\right)=\mathbb{E}^{x_{n}}\left[e^{-\int_{0}^{t} \frac{n(n-2)}{4} d s} f\left(X_{n}(t)\right)\right] .
$$

Hence, if $X_{n}^{*}(0)=z>0$, we have

$$
T_{t}^{*} h(z)=e^{-n(n-2) t / 4} \mathbb{E}\left[\left(z e^{W_{t}-t}\right)^{1-\frac{n}{2}}\right]=h(z) e^{-n(n-2) t / 4} \mathbb{E}\left[e^{\left(1-\frac{n}{2}\right) W_{t}-\left(1-\frac{n}{2}\right) t}\right]=h(z),
$$

since

$$
\mathbb{E}\left[e^{\left(1-\frac{n}{2}\right) W_{t}}\right]=e^{t\left(1-\frac{n}{2}\right)^{2}}
$$

Denote by $\mathbf{X}^{h}(t)$ the process we get from $\mathbf{X}^{*}(t)$ by using Doob's $h$-transform with $h\left(x_{n}\right)=$ $x_{n}^{1-\frac{n}{2}}$. Because $h$ is invariant for $\mathbf{X}^{*}$, then $\mathbb{P}\left(\mathbf{X}^{h}(t) \in \mathbb{H}^{n}\right)=1$ for all $t>0$.

Recall that (see e.g. [19], page 357) if $\mathcal{G}$ is a generator of a diffusion and $h$ is a positive function, harmonic for $\mathcal{G}$, then the generator $\mathcal{G}^{h}$ of an $h$-process of this diffusion is given by the following formula: for $f \in C^{2}$ with compact support

$$
\mathcal{G}^{h} f(x)=\frac{1}{h(x)} \mathcal{G}(h f)(x) .
$$

In this way, starting from an Euclidean Brownian motion in a $\mathbb{H}^{n} \subset \mathbb{R}^{n}$ and using successively a time-change, killing and Doob's $h$-transform, we get a process $\mathbf{X}^{h}$ with the following generator

$$
\begin{gathered}
L_{2}^{h} f(x)=x_{n}^{\frac{n}{2}-1}\left(x_{n}^{2} \Delta\left(x_{n}^{1-\frac{n}{2}} f(x)\right)-\frac{n(n-2)}{4} x_{n}^{1-\frac{n}{2}} f(x)\right) \\
=x_{n}^{2} \Delta f(x)-(n-2) x_{n} \frac{\partial f}{\partial x_{n}} .
\end{gathered}
$$

Thus for $f \in C^{2}\left(\mathbb{H}^{n}\right)$ with compact support the formula describing $L_{2}^{h}$ is the same as the formula for $\Delta_{\mathbb{H}^{n}}$ so that the process we have just constructed has the same law as a hyperbolic Brownian motion in $\mathbb{H}^{n}$. 
Now we recall how a new clock and Doob's $h$-transform change the Green function and the harmonic measure (or Poisson kernel) of a domain $D$. We start with a relation between Green functions of a process $Z(t)$ and of its time-change.

Let $G_{D}$ be the Green operator for a domain $D$ and process $Z(t)$ killed on exiting $D$, $\tau_{D}=\inf \{t>0: Z(t) \notin D\}$, and let $G_{D}(x, y)$ be the kernel of $G_{D}$. Then

$$
G_{D} f(x)=\mathbb{E}^{x} \int_{0}^{\tau_{D}} f\left(Z_{s}\right) d s=\int G_{D}(x, y) f(y) d y .
$$

Now let us consider the same process $Z(t)$ as above but with time-change. Let $\xi$ be a strictly positive continuous function and define a functional $A(t)=\int_{0}^{t} \xi\left(Z_{s}\right) d s$. Now take its inverse $A^{-1}(t)$ and consider a process $Y(t)=Z\left(A^{-1}(t)\right)$, killed at the moment $\tau_{D}^{Y}$ of exiting $D$. Since

$$
\tau_{D}^{Y}=\inf \{t: Y(t) \notin D\}=\inf \{A(t): Z(t) \notin D\}=A\left(\tau_{D}\right),
$$

hence for Borel and bounded function $f$,

$$
\begin{aligned}
G_{D} f(x) & =\mathbb{E}^{x}\left[\int_{0}^{\tau_{D}^{Y}} f(Y(t)) d t\right]=\mathbb{E}^{x}\left[\int_{0}^{A^{-1}\left(A\left(\tau_{D}\right)\right)} f(Z(s)) \xi(Z(s)) d s\right] \\
& =\int G_{D}(x, y) \xi(y) f(y) d y,
\end{aligned}
$$

which means that

$$
G_{D}^{Y}(x, y)=\xi(y) G_{D}(x, y) .
$$

In particular, let $Z(t)$ be a process with generator $\Delta$ and Green function $G_{D}^{\Delta}(x, y)$, and let a process $Y(t)$ have a generator $L_{1}=x_{n}^{2} \Delta$ and a Green function $G_{D}^{L_{1}}$. If we put $A(t)=$ $\int_{0}^{t} Z_{n}^{-2}(s) d s$, then $Y(t)=Z\left(A^{-1}(t)\right)$ in law. Then, for $D \subset \mathbb{H}^{n}$,

$$
G_{D}^{L_{1}}(x, y)=\frac{1}{y_{n}^{2}} G_{D}^{\Delta}(x, y), x, y \in \mathbb{H}^{n} .
$$

Now we turn our attention to harmonic measures. Let $D \subset \mathbb{H}^{n}$ be such domain that $\inf \left\{z_{n}: z \in D\right\}>0$. Then for $A \subset \partial D$ we have $\omega_{D}(x, A)=\mathbb{P}^{x}\left(X\left(\tau_{D}\right) \in A\right)$. If $\omega_{D}(x, \cdot)$ is absolutely continuous with respect to a reference measure on $\partial D$, the density of $\omega_{D}(x, \cdot)$ is called the Poisson kernel of a process $X$ and domain $D$. Observe that a time-change does not change points hit by the process exiting from $D$. This means that the harmonic measure for time-changed process is the same as the harmonic measure for the initial process.

Recall how the Green function and the harmonic measure of an open set are changed under an $h$-transform of the process. By Eqs. 7 and 5 the Green operator for the $h$-process is given by

$G_{D}^{h} f(x)=\frac{1}{h(x)} \mathbb{E}^{x} \int_{0}^{\tau_{D}} h\left(Z_{t}\right) f\left(Z_{t}\right) d t=\frac{1}{h(x)} \int G_{D}(x, y) h(y) f(y) d y, \quad$ for $x \in \mathbb{H}^{n}$.

Keeping in mind that our harmonic function $h$ is finite and strictly positive on $\mathbb{H}^{n}$, we infer that if $p_{t}(x, y)$ is a transition density of a process $Z$ then the transition density for the $h$-process is given by the following formula

$$
p_{t}^{h}(x, y)=\frac{h(y)}{h(x)} p_{t}(x, y) .
$$


Let $A \subset \partial D$. Then by the very definition of a harmonic measure, using paths counting yields the following formula

$$
\omega_{D}(x, A)=\mathbb{P}^{x}\left(Z\left(\tau_{D}\right) \in A\right)=\int_{0}^{\infty} p_{t}(x, A) \mathbb{P}^{x}\left(\tau_{D} \in d t ; X(s) \in D, 0<s<\tau_{D}\right),
$$

so that

$$
\begin{aligned}
\omega_{D}^{h}(x, d y) & =\mathbb{P}^{x}\left(Z^{h}\left(\tau_{D}\right) \in d y\right)=\int_{0}^{\infty} p_{t}^{h}(x, d y) \mathbb{P}^{x}\left(\tau_{D} \in d t ; Z(s) \in D, 0<s<\tau_{D}\right) \\
& =\frac{h(y)}{h(x)} \omega_{D}(x, d y) .
\end{aligned}
$$

From now on we will denote by $G_{D}(x, y)$ the Green function of $D$ (with respect to the hyperbolic reference measure, that is with respect to the Riemannian measure $d \mu$ ) of the hyperbolic Brownian motion $\mathbf{B}$ and by $G_{D}^{L}(x, y)$ the Green function of $D$ corresponding to the operator

$$
L=\Delta-q_{1}
$$

with the Lebesgue measure as the reference measure. Recall that $q_{1}(x)=\frac{n(n-2)}{4 x_{n}^{2}}$. By $\omega_{D}(x, d y)$ and $\omega_{D}^{L}(x, d y)$ we denote the harmonic measures corresponding to the hyperbolic Brownian motion or to the operator $L$, respectively. Let $G_{D}^{\Delta}(x, y)$ and $\omega_{D}^{\Delta}(x, d y)$ denote the Green function and the harmonic measure for the Euclidean Brownian motion, killed on exiting $D$. Below we list the crucial relationships between these objects. From now on we always denote $h(x)=x_{n}^{1-\frac{n}{2}}, x \in \mathbb{H}^{n}$.

Theorem 4.1 For any open set $D \subseteq \mathbb{H}^{n}$ we have for $x, y \in D$,

$$
\begin{aligned}
G_{D}(x, y) & =\frac{1}{h(x) h(y)} G_{D}^{L}(x, y) \\
& \leq \frac{1}{h(x) h(y)} G_{D}^{\Delta}(x, y) .
\end{aligned}
$$

Let $a>0$. For any open set $D \subseteq\left\{z_{n}>a\right\} \subset \mathbb{H}^{n}$ we have for $x, \in D, y \in \partial D$

$$
\begin{aligned}
\omega_{D}(x, d y) & =\frac{h(y)}{h(x)} \omega_{D}^{L}(x, d y) \\
& \leq \frac{h(y)}{h(x)} \omega_{D}^{\Delta}(x, d y) .
\end{aligned}
$$

Proof Recall that $\Delta_{\mathbb{H}^{n}}=\left(x_{n}^{2} L\right)^{h}$. Let $G_{D}^{*}(x, y)$ be the hyperbolic Green function of $D$ with the Lebesgue measure as the reference measure. We apply (8) with $\xi(x)=\frac{1}{x_{n}^{2}}$ to obtain

$$
G_{D}^{*}(x, y)=\frac{1}{y_{n}^{2}} G_{D}^{L^{h}}(x, y)=\frac{1}{y_{n}^{2}} \frac{h(y)}{h(x)} G_{D}^{L}(x, y)=\frac{1}{h(y) h(x)} G_{D}^{L}(x, y) y_{n}^{-n} .
$$

The equality holds whenever the Green function $G_{D}^{L}(x, y)$ exists and in fact holds for any $D$. Since $G_{D}(x, y)=G_{D}^{*}(x, y) y_{n}^{-n}$ we obtain (9). But the process corresponding to the operator $L$ can be realized by killing the trajectories of the ordinary Brownian motion, so that by Eq. 9, we obtain (10).

Next, we deal with the harmonic measure. Since $\left(x_{n}^{2} L\right)^{h}=\Delta_{\mathbb{H}^{n}}$, the harmonic measure of $D$ for $\Delta_{\mathbb{H} n}$ is the same as for $L^{h}$ and the latter is the $h$-transform of the harmonic measure of $D$ for $L$, which proves (11). The same argument as for (10) can be employed to show (12). 
As a consequence, we can explore the potential theory of the hyperbolic Laplacian via the potential theory for the generator $L$. The generator $L$ can be studied using general results on Schrödinger perturbations of the Laplacian, that is operators of the form $\Delta-q$, where the real function $q$ is from the so called Kato class [9]. In our particular case $q(x)=q_{1}(x)=$ $\frac{n(n-2)}{4 x_{n}^{2}}$. To deal with the Green function $G_{D}^{L}$ corresponding to the generator $L$, we use the results of the paper [24]. Let the measure $\mathbb{P}_{y}^{x}, x \in D, y \in D$, be the distribution of the Doob $G_{D}^{\Delta}(\cdot, y)$-transform of the Euclidean Brownian motion. By $\mathbb{E}_{y}^{x}$ we denote the corresponding expectation for $x \neq y, x, y \in D$. Then the transition densities of the Doob transform are given by

$$
p_{D}^{y}(t ; x, u)=p_{D}^{\Delta}(t ; x, u) \frac{G_{D}^{\Delta}(u, y)}{G_{D}^{\Delta}(x, y)}, u \in D,
$$

where $p_{D}^{\Delta}(t ; \cdot, \cdot)$ is the transition density corresponding to the Euclidean Brownian motion.

The main result of [24] implies that for any $C^{1,1}$ bounded set $D \subseteq \mathbb{H}^{n}$ we have

$$
G_{D}^{L}(x, y)=G_{D}^{\Delta}(x, y) n_{D}(x, y), x, y \in D,
$$

where the conditional gauge function $n_{D}$ is defined by

$$
n_{D}(x, y)=\mathbb{E}_{y}^{x} \exp \left(-\int_{0}^{\tau_{D}} \frac{n(n-2)}{4 W_{n}^{2}(t)} d t\right), x, y \in D .
$$

The next crucial result of [24] is about the harmonic measure of $D$ for $L$. Namely, it is absolutely continuous with respect to the Euclidean surface measure on $\partial D$ and its density, called the Poisson kernel, is given by

$$
P_{D}^{L}(x, z)=P_{D}^{\Delta}(x, z) n_{D}(x, z), \quad x \in D, z \in \partial D .
$$

where, for $x \in D, z \in \partial D, n_{D}(x, z)$ is the value at $z$ of the continuous extension of $n_{D}(x, \cdot)$ to $\partial D$, existing by the results of [24]. Here $P_{D}^{\Delta}(x, z)$ is the Poisson kernel of $D$, corresponding to the Laplacian $\Delta$.

Corollary 4.2 Let $D \subseteq \mathbb{H}^{n}$ be an open $C^{1,1}$ bounded set. For $x, y \in D$ we have

$$
G_{D}(x, y)=\frac{1}{h(x) h(y)} n_{D}(x, y) G_{D}^{\Delta}(x, y) .
$$

If $x \in D$ and $z \in \partial D$ then the hyperbolic Poisson kernel $P_{D}(x, z)$ is given as

$$
\begin{aligned}
P_{D}(x, z) & =\frac{h(z)}{h(x)} P_{D}^{\Delta}(x, z) n_{D}(x, z) \\
& =h^{2}(z) \frac{\partial G_{D}(x, \cdot)}{\partial n_{z}},
\end{aligned}
$$

where $n_{z}$ is the inward unit vector normal to $\partial D$ at $z$ and the directional derivative is computed at $z$. Here $P_{D}(x, z)$ is understood as the density of the hyperbolic harmonic measure of $D$ with respect to the classical surface measure on $\partial D$.

Proof Except for the last equality everything follows from Theorem 4.1 together with (13) and (14). Next, by (15) and continuity of $n_{D}(x, y)$ with respect to $y \in \bar{D}$, we have

$$
P_{D}^{\Delta}(x, z)=\frac{\partial G_{D}^{\Delta}(x, \cdot)}{\partial n_{z}}=\frac{h(z) h(x)}{n_{D}(x, z)} \frac{\partial G_{D}(x, y)}{\partial n_{z}},
$$


which together with (16) yields

$$
P_{D}(x, z)=h^{2}(z) \frac{\partial G_{D}(x, \cdot)}{\partial n_{z}} .
$$

\section{Hyperbolic Green Function Estimates}

In this section we will employ Theorem 4.1 to derive general estimates for the Green function $G_{D}$ of a smooth open set $D \subset \mathbb{H}^{n}$.

Let $U(x, y)$ be the potential kernel (with respect to the hyperbolic reference measure). It is shown in [4] that

$$
U(x, y) \approx\left(\frac{x_{n} y_{n}}{|x-y|^{2}}\right)^{\frac{n}{2}-1}\left(1 \wedge \frac{x_{n} y_{n}}{|x-y|^{2}}\right)^{\frac{n}{2}} .
$$

Rewriting the formula (4) into the form

$$
2 \frac{x_{n} y_{n}}{|x-y|^{2}}=\frac{1}{\cosh d_{\mathbb{H}^{n}}(x, y)-1},
$$

we conclude

$$
U(x, y) \approx \frac{1}{\left(\cosh d_{\mathbb{H}^{n}}(x, y)-1\right)^{\frac{n}{2}-1}\left(\cosh d_{\mathbb{H}^{n}}(x, y)\right)^{\frac{n}{2}}} \approx \frac{1}{\left(1 \wedge d_{\mathbb{H}^{n}}(x, y)\right)^{n-2} e^{(n-1) d_{\mathbb{H}^{n}}(x, y)}} .
$$

Equivalently, we may write

$$
U(x, y) \approx\left\{\begin{array}{l}
\frac{1}{\left(d_{\mathbb{H}^{n}}(x, y)\right)^{n-2}}, d_{\mathbb{H}^{n}}(x, y) \leq 1, \\
\frac{1}{\left(\cosh d_{\mathbb{H}^{n}}(x, y)\right)^{n-1}}, d_{\mathbb{H}^{n}}(x, y) \geq 1 .
\end{array}\right.
$$

Let us recall that by $B(x, R), x \in \mathbb{H}^{n}, R>0$, we denote the hyperbolic ball of radius $R$ and center $x$. Let $e_{n}=(0, \ldots, 0,1)$. To shorten notation, we will denote the ball $B\left(e_{n}, R\right)$ by $B_{R}$. For any two balls of the same radius there is an isometry mapping one ball onto another, so we can always work with a ball with a conveniently picked center and usually the center is either $e_{n}$ or $e_{n}(R)=\left(0, \ldots, 0, e^{R}\right), R>0$. Note that $d_{\mathbb{H}^{n}}\left(e_{n}, e_{n}(R)\right)=R$.

As a subset of Euclidean space $\mathbb{R}^{n}, B_{R}$ is a ball of radius $\sinh R$ centered at $(0, \ldots, 0, \cosh R)$, i.e. it is given by the inequality

$$
|\tilde{x}|^{2}+\left(\cosh R-x_{n}\right)^{2}<\sinh ^{2} R,
$$

where $\tilde{x}=\left(x_{1}, \ldots, x_{n-1}\right)$.

We will say that an open set $D \subset \mathbb{H}^{n}$ is $C^{1,1}$ with hyperbolic characteristic $r_{0}>0$ if for any point $z \in \partial D$ there are two hyperbolic balls $B^{(1)}(z) \subseteq D$ and $B^{(2)}(z) \subseteq \mathbb{H}^{n} \backslash D$ of radius $r_{0}$ such that $\overline{B^{(1)}(z)} \cap \overline{B^{(2)}(z)}=\{z\}$. Note that, if $D$ has a hyperbolic characteristic $r_{0}>0$, then it has also a hyperbolic characteristic $r_{0}^{\prime}>0$ for any $r_{0}^{\prime}<r_{0}$. For example any hyperbolic ball of radius $R$ has a characteristic $R$, but also $r<R$.

The Euclidean characteristic is defined in a similar way. If an open set D is contained in a strip $0<a<z_{n}<b$, then it is clear that Euclidean and hyperbolic characteristics of $D$ are comparable and the comparability constant depends on $a$ and $b / a$. For a set $D \subset$ $\mathbb{H}^{n}$ we denote by $\operatorname{diam}_{H} D$ and $\operatorname{diam}_{E} D$ its diameters in hyperbolic and Euclidean metric, respectively. It is clear that if $\operatorname{diam}_{H} D<\infty$ then $\operatorname{diam}_{E} D<\infty$. 
To get more precise two sided estimates of $G_{D}(x, y)$ we need to evaluate the conditional gauge function $n_{D}$. The exact computation of $n_{D}$ seems to be a very difficult task, but we can find some simple estimates which by no means are sharp but will be helpful later on.

Lemma 5.1 Assume that $D \subset\left\{z: z_{n} \geq a\right\}$ is a bounded $C^{1,1}$ domain with Euclidean characteristic $r_{0}>0$. There is $C^{*}=C^{*}\left(n, \frac{\operatorname{diam}_{E} D}{r_{0}}, \frac{\operatorname{diam}_{E} D}{a}\right)$ such that

$$
n_{D}(x, y) \geq C^{*}, x, y \in D \text {. }
$$

Proof Let $x, y \in D$. Observing that $W_{n}^{2}(t) \geq a^{2}$ if $t \leq \tau_{D}$, we have

$$
\mathbb{E}_{y}^{x} \int_{0}^{\tau_{D}} \frac{n(n-2)}{4 W_{n}^{2}(t)} d t \leq \frac{n(n-2)}{4 a^{2}} \mathbb{E}_{y}^{x} \tau_{D}
$$

By Jensen's inequality

$$
\begin{aligned}
\mathbb{E}_{y}^{x}\left[\exp \left(-\int_{0}^{\tau_{D}} \frac{n(n-2)}{4 W_{n}^{2}(t)} d t\right)\right] & \geq \exp \left(-\mathbb{E}_{y}^{x} \int_{0}^{\tau_{D}} \frac{n(n-2)}{4 W_{n}^{2}(t)} d t\right) \\
& \geq \exp \left(-\frac{n(n-2)}{4 a^{2}} \mathbb{E}_{y}^{x} \tau_{D}\right) .
\end{aligned}
$$

By virtue of $3 G$-theorem of Cranston-Fabes-Zhao [11], we get

$$
\begin{aligned}
\mathbb{E}_{y}^{x} \tau_{D} & =\int_{D} \frac{G_{D}^{\Delta}(x, z) G_{D}^{\Delta}(z, y)}{G_{D}^{\Delta}(x, y)} d z \\
& \leq C\left(n, \operatorname{diam}_{E} D, r_{0}\right) .
\end{aligned}
$$

This and the scaling property

$$
\mathbb{E}_{w}^{u} \tau_{r D}=r^{2} \mathbb{E}_{w / r}^{u / r} \tau_{D}, \quad r>0 ; \quad u, w \in r D
$$

yield

$$
\mathbb{E}_{y}^{x} \tau_{D} \leq C\left(n, \frac{\operatorname{diam}_{E} D}{r_{0}}\right) \times\left(\operatorname{diam}_{E} D\right)^{2},
$$

where $C=C\left(n, \frac{\operatorname{diam}_{E} D}{r_{0}}\right)$ is increasing with respect to the second variable. The conclusion follows from (19).

The above lemma, Corollary 4.2 and Theorem 4.1 imply the following corollary.

Corollary 5.2 Let $D \subset \mathbb{H}^{n}$ be a bounded $C^{1,1}$ domain with the Euclidean characteristic $r_{0}>0$ contained in the halfspace $z_{n}>a>0$. There is a constant $C^{*}=$ $C^{*}\left(n, \frac{\operatorname{diam}_{E} D}{r_{0}}, \frac{\operatorname{diam}_{E} D}{a}\right)$ such that

$$
C^{*} G_{D}^{\Delta}(x, y) \leq\left(x_{n} y_{n}\right)^{\frac{n}{2}-1} G_{D}(x, y) \leq G_{D}^{\Delta}(x, y), x, y \in D
$$

and

$$
C^{*} \mathbb{P}^{x}\left(\boldsymbol{W}\left(\tau_{D}\right) \in d z\right) \leq\left(\frac{z_{n}}{x_{n}}\right)^{\frac{n}{2}-1} \mathbb{P}^{x}\left(\mathbf{B}\left(\tau_{D}\right) \in d z\right) \leq \mathbb{P}^{x}\left(\boldsymbol{W}\left(\tau_{D}\right) \in d z\right), x, z \in \bar{D} .
$$

In particular for $B_{R}$, the hyperbolic ball of radius $R$ and centered at $e_{n}$,

$$
\begin{gathered}
\frac{1}{C_{R}}\left(x_{n} y_{n}\right)^{1-\frac{n}{2}} G_{B_{R}}^{\Delta}(x, y) \leq G_{B_{R}}(x, y) \leq\left(x_{n} y_{n}\right)^{1-\frac{n}{2}} G_{B_{R}}^{\Delta}(x, y), x, y \in B_{R}, \\
\frac{1}{C_{R}}\left(\frac{x_{n}}{z_{n}}\right)^{\frac{n}{2}-1} P_{B_{R}}^{\Delta}(x, z) \leq P_{B_{R}}(x, z) \leq\left(\frac{x_{n}}{z_{n}}\right)^{\frac{n}{2}-1} P_{B_{R}}^{\Delta}(x, z), x \in B_{R}, z \in \partial B_{R},
\end{gathered}
$$


where $1 \leq C_{R}<\infty$ and $C_{R}$ increases with $R$. We recall that $P_{B_{R}}$ is the hyperbolic Poisson kernel with respect to the Euclidean surface measure on $\partial B_{R}$.

For any set $D \subset \mathbb{H}^{n}$ and $x \in \mathbb{H}^{n}, \delta_{D}(x)$ denotes the hyperbolic distance from $x$ to $\partial D$.

Proposition 5.3 Let $D$ be a $C^{1,1}$ set with the hyperbolic characteristic $0<r_{0} \leq 1$. Let $x \in D$ and $Q \in \partial D$ be such that $d(x, Q) \leq r_{0} / 4$. There is a constant $C=C(n)$ such that

$$
C^{-1} \frac{\delta_{D}(x)}{r_{0}} \leq \mathbb{P}^{x}\left(\mathbf{B}\left(\tau_{D \cap B\left(Q, r_{0}\right)}\right) \notin \partial D\right) \leq C \frac{\delta_{D}(x)}{r_{0}} .
$$

Proof We may assume that $Q=e_{n}$. Let $x \in D \cap B\left(Q, r_{0} / 4\right)$. There is a ball $B\left(Q_{x}, r_{0}\right) \subset$ $D, Q_{x} \in D, x \in B\left(Q_{x}, r_{0}\right)$, such that $\delta_{D}(x)=\delta_{B\left(Q_{x}, r_{0}\right)}(x)$. Let $S=B\left(Q_{x}, r_{0}\right) \cap$ $\partial B\left(Q, r_{0}\right) \subset D$ and $S_{1}=\partial B\left(Q_{x}, r_{0}\right) \backslash B\left(Q, r_{0}\right)$. If the process exits $B\left(Q_{x}, r_{0}\right)$ through $S_{1}$ it must exit $D \cap B\left(Q, r_{0}\right)$ through $S$, hence

$$
\mathbb{P}^{x}\left(\mathbf{B}\left(\tau_{D \cap B\left(Q, r_{0}\right)}\right) \notin \partial D\right) \geq \mathbb{P}^{x}\left(\mathbf{B}\left(\tau_{B\left(Q_{x}, r_{0}\right)}\right) \in S_{1}\right) .
$$

Since $d_{\mathbb{H}^{n}}\left(Q_{x}, Q\right) \geq d_{\mathbb{H}^{n}}\left(Q_{x}, x\right)-d_{\mathbb{H}^{n}}(Q, x)=r_{0}-\delta_{D}(x)-d_{\mathbb{H}^{n}}(Q, x) \geq r_{0} / 2$, the (Euclidean) surface measure of $S_{1}$ is at least $c_{1} r_{0}^{n-1}$, where $c_{1}=c_{1}(n)$. Hence, by Corollary 5.2 and the formula for the classical Poisson kernel of a ball, we obtain

$$
\mathbb{P}^{x}\left(\mathbf{B}\left(\tau_{B\left(Q_{x}, r_{0}\right)}\right) \in S_{1}\right) \geq c_{2}(n) \frac{\delta_{D}(x)}{r_{0}} .
$$

In the above proof we used the fact that in the ball $B\left(e_{n}, 2\right)$ the hyperbolic and Euclidean distances are equivalent.

To prove the upper bound we observe that there is an outer ball $B\left(Q_{x}^{\prime}, r_{0} / 8\right)$ contained in $D^{c}$ such that $\delta_{B^{c}}\left(Q_{x}^{\prime}, r_{0} / 8\right)(x)=\delta_{D}(x)$. Let $z$ be such that $d_{\mathbb{H}^{n}}\left(z, Q_{x}^{\prime}\right) \leq r_{0} / 3$ then $d_{\mathbb{H}^{n}}(z, Q) \leq d_{\mathbb{H}^{n}}\left(z, Q_{x}^{\prime}\right)+d_{\mathbb{H}^{n}}\left(x, Q_{x}^{\prime}\right)+d_{\mathbb{H}^{n}}(Q, x) \leq r_{0}$. It follows that $B\left(Q_{x}^{\prime}, r_{0} / 3\right) \subset$ $B\left(Q, r_{0}\right)$ and

$$
B\left(Q_{x}^{\prime}, r_{0} / 3\right) \backslash B\left(Q_{x}^{\prime}, r_{0} / 8\right) \subset B\left(Q, r_{0}\right) \backslash B\left(Q_{x}^{\prime}, r_{0} / 8\right),
$$

hence

$$
\begin{aligned}
\mathbb{P}^{x}\left(\mathbf{B}\left(\tau_{D \cap B\left(Q, r_{0}\right)}\right) \notin \partial D\right) & \leq \mathbb{P}^{x}\left(\mathbf{B}\left(\tau_{B\left(Q_{x}^{\prime}, r_{0} / 3\right) \backslash B\left(Q_{x}^{\prime}, r_{0} / 8\right)}\right) \in \partial B\left(Q_{x}^{\prime}, r_{0} / 3\right)\right) \\
& \leq c \delta_{D}(x) / r_{0},
\end{aligned}
$$

where the last estimate follows from [8].

The following lemma can be proved exactly in the same way as the lower bound in Proposition 5.3.

Lemma 5.4 Let $x_{0} \in \partial B\left(y_{0}, R\right)$. Let $0<M \leq R / 2$. Suppose that $S=\left\{z \in B\left(y_{0}, R\right)\right.$ : $\left.d_{\mathbb{H}^{n}}\left(z, x_{0}\right)=2 M, \delta_{B\left(y_{0}, R\right)}(z) \geq M\right\}$. Let $x \in B\left(y_{0}, R\right)$ be such that $d_{\mathbb{H}^{n}}\left(x, x_{0}\right)=$ $\delta_{B\left(y_{0}, R\right)}(x) \leq M$. Then there is a constant $C=C(n, M)$ such that

$$
\mathbb{P}^{x}\left(\mathbf{B}\left(\tau_{D}\right) \in S\right) \geq C \delta_{B\left(y_{0}, R\right)}(x),
$$

where $D=B\left(y_{0}, R\right) \cap B\left(x_{0}, 2 M\right)$.

Lemma 5.5 Let $0<r_{0} \leq 1$. There exist $0<\kappa<1$ and $A \geq 1$ such that, if $D$ is a $C^{1,1}$ set with the hyperbolic characteristic $r_{0}$, then for $Q \in \partial D$ there is a $C^{1,1}$ domain $F \subset D$, with 
hyperbolic characteristic $\kappa r_{0}$ such that $F \subset B\left(Q, A r_{0}\right)$, and

$$
F \cap B(Q, r / 4)=D \cap B(Q, r / 4) .
$$

The proof of this Lemma in the Euclidean case is contained in [3]. Since, by standard argument, we may assume that $Q=e_{n}$ and the hyperbolic and the Euclidean distances are equivalent in any ball with center $e_{n}$, the same proof will work in our case.

Lemma 5.6 Let $D \subset B\left(e_{n}, \kappa r_{0}\right)$ be a $C^{1,1}$ connected domain with the hyperbolic characteristic $r_{0}$, where $r_{0} \leq 1 \leq \kappa$. There is a constant $C=C(n, \kappa)$, such that for $x, y \in D$

$$
C^{-1}\left(1 \wedge \frac{\delta_{D}(x) \delta_{D}(y)}{d_{\mathbb{H}^{n}}(x, y)^{2}}\right) U(x, y) \leq G_{D}(x, y) \leq C\left(1 \wedge \frac{\delta_{D}(x) \delta_{D}(y)}{d_{\mathbb{H}^{n}}(x, y)^{2}}\right) U(x, y) .
$$

This follows from Corollary 5.2 and the estimates of the Green function for the Brownian motion for $C^{1,1}$ domains, where the constant depends on the domain through the ratio $\operatorname{diam}_{E} D / r_{0}$. Also we use the fact that the Euclidean and hyperbolic distances are comparable in $B\left(e_{n}, \kappa r_{0}\right)$ with the comparability constant dependent on $n$ and $\kappa$. We refer to the results obtained in the paper [2] which should be combined with the observation that the Lipschitz constant in the definition of the boundary of $D$ can be bounded by an absolute constant.

The following theorem is an improvement of the upper bound of the Green function given in Corollary 5.2 and is the main result of this section.

Theorem 5.7 Let $0<r_{0} \leq 1$ and let $D$ be a $C^{1,1}$ set with the hyperbolic characteristic $r_{0}$. Then there is a constant $C=C(n)$ such that

$$
G_{D}(x, y) \leq C\left(1 \wedge \frac{\left(r_{0} \wedge \delta_{D}(x)\right)\left(r_{0} \wedge \delta_{D}(y)\right)}{r_{0}^{2} \wedge d_{\mathbb{H}^{n}}(x, y)^{2}}\right) U(x, y), x, y \in D
$$

Proof By symmetry of $G_{D}$ we can assume that $\delta_{D}(x) \leq \delta_{D}(y)<\infty$. By a standard argument, we can assume that the point $e_{n} \in \partial D$ and $d_{\mathbb{H}^{n}}\left(e_{n}, x\right)=\delta_{D}(x)$.

If $\delta_{D}(x)>r_{0} / 512$, then the upper bound holds trivially, since $G_{D}(x, y) \leq U(x, y)$. Let $\delta_{D}(x) \leq r_{0} / 512$. Suppose that $d_{\mathbb{H}^{n}}(x, y) \geq r_{0} / 32$. Observe that for $z \in \overline{B\left(e_{n}, r_{0} / 128\right)}$,

$$
\left|d_{\mathbb{H}^{n}}(z, y)-d_{\mathbb{H}^{n}}(x, y)\right| \leq d_{\mathbb{H}^{n}}\left(z, e_{n}\right)+d_{\mathbb{H}^{n}}\left(x, e_{n}\right) \leq \frac{r_{0}}{64} .
$$

Hence, by (17),

$$
\sup _{\left\{z: d_{\mathbb{H}^{n}}\left(z, e_{n}\right)=r_{0} / 128\right\}} U(z, y) \leq C(n) U(x, y) .
$$

Let $F=D \cap B\left(e_{n}, r_{0} / 128\right)$. By Proposition 5.3, we have

$$
\mathbb{P}^{x}\left(d_{\mathbb{H}^{n}}\left(\mathbf{B}\left(\tau_{F}\right), e_{n}\right)=r_{0} / 128\right)=\mathbb{P}^{x}\left(\mathbf{B}\left(\tau_{F}\right) \notin \partial D\right) \leq C(n) \frac{\delta_{D}(x)}{r_{0}},
$$


so that we arrive at (note that $\left.d_{\mathbb{H}^{n}}\left(e_{n}, y\right) \geq d_{\mathbb{H}^{n}}(x, y)-d_{\mathbb{H}^{n}}\left(e_{n}, x\right) \geq \frac{1}{32} r_{0}-\frac{1}{64} r_{0} \geq \frac{r_{0}}{64}\right)$

$$
\begin{aligned}
G_{D}(x, y) & =\mathbb{E}^{x} G_{D}\left(\mathbf{B}\left(\tau_{F}\right), y\right) \\
& \leq \sup _{\left\{z: d_{\mathbb{H}^{n}}\left(z, e_{n}\right)=r_{0} / 128\right\}} U(z, y) \mathbb{P}^{x}\left(d_{\mathbb{H}^{n}}\left(\mathbf{B}\left(\tau_{F}\right), e_{n}\right)=r_{0} / 128\right) \\
& \leq C U(x, y) \frac{\delta_{D}(x)}{r_{0}} .
\end{aligned}
$$

If $d_{\mathbb{H}^{n}}(x, y)>r_{0} / 16, \delta_{D}(x) \leq r_{0} / 512, \delta_{D}(y) \leq r_{0} / 512$, and $z$ is such that $d_{\mathbb{H}^{n}}\left(z, e_{n}\right)=$ $r_{0} / 128$, then

$$
d_{\mathbb{H}^{n}}(z, y) \geq d_{\mathbb{H}^{n}}(x, y)-d_{\mathbb{H}^{n}}\left(z, e_{n}\right)-d_{\mathbb{H}^{n}}\left(x, e_{n}\right) \geq r_{0} / 16-r_{0} / 128-r_{0} / 512 \geq r_{0} / 32 .
$$

Repeating the argument used to derive Eq. 20, for $d_{\mathbb{H} \mathbb{H}^{n}}(x, y)>r_{0} / 16, \delta_{D}(x) \leq r_{0} / 64$ and $\delta_{D}(y) \leq r_{0} / 64$, we obtain, by Eq. 20,

$$
\begin{aligned}
G_{D}(x, y) & =\mathbb{E}^{x} G_{D}\left(\mathbf{B}\left(\tau_{F}\right), y\right) \\
& \leq \sup _{\left\{z \in D: d_{\mathbb{H}^{n}}\left(z, e_{n}\right)=r_{0} / 128\right\}} G_{D}(z, y) \mathbb{P}^{x}\left(d_{\mathbb{H}^{n}}\left(\mathbf{B}\left(\tau_{F}\right), e_{n}\right)=r_{0}\right) \\
& \leq C \sup _{\left\{z: d_{\mathbb{H}^{n}}\left(z, e_{n}\right)=r_{0} / 128\right\}} U(z, y) \frac{\delta_{D}(y)}{r_{0}} \frac{\delta_{D}(x)}{r_{0}} \\
& \leq C U(x, y) \frac{\delta_{D}(x) \delta_{D}(y)}{r_{0}^{2}}
\end{aligned}
$$

with a constant $C=C(n)$. Hence if $d_{\mathbb{H}^{n}}(x, y)>r_{0} / 16$, regardless how close the points are to the boundary, we have

$$
G_{D}(x, y) \leq C\left(1 \wedge \frac{\left(r_{0} \wedge \delta_{D}(x)\right)\left(r_{0} \wedge \delta_{D}(y)\right)}{r_{0}^{2} \wedge d_{\mathbb{H}^{n}}(x, y)^{2}}\right) U(x, y),
$$

with $C=C(n)$.

Now, let $d_{\mathbb{H}^{n}}(x, y) \leq r_{0} / 16$ and $\delta_{D}(x) \leq r_{0} / 16$. This implies that $\delta_{D}(y) \leq r_{0} / 8$. By Lemma 5.5 there are positive $\kappa<1$ and $A \geq 1$ such that we can construct a $C^{1,1}$ set $D^{*}$ having the hyperbolic characteristic $\kappa r_{0}$ satisfying

$$
B\left(e_{n}, r_{0} / 4\right) \cap D \subset D^{*} \subset B\left(e_{n}, A r_{0}\right) \cap D .
$$

Since $d_{\mathbb{H}^{n}}(x, y) \leq r_{0} / 16$, then $x, y \in D^{*}$. Moreover, $\delta_{D}(x) \leq \delta_{D}(y) \leq r_{0} / 8$ implies that $\delta_{D}(x)=\delta_{D^{*}}(x)$ and $\delta_{D}(y)=\delta_{D^{*}}(y)$. We also note that $\partial D^{*}$ and $\partial D$ coincide in the ball $B\left(e_{n}, r_{0} / 4\right)$, hence

$$
\partial D^{*} \backslash \partial D \subset B\left(e_{n}, r_{0} / 4\right)^{c} .
$$

This implies, by Proposition 5.3,

$$
\mathbb{P}^{x}\left(\mathbf{B}\left(\tau_{D^{*}}\right) \in \partial D^{*} \backslash \partial D\right) \leq \mathbb{P}^{x}\left(\mathbf{B}\left(\tau_{D \cap B\left(e_{n}, r_{0} / 4\right)}\right) \notin B\left(e_{n}, r_{0} / 4\right)\right) \leq C \frac{\delta_{D}(x)}{r_{0}},
$$

with $C=C(n)$. By the strong Markov property,

$$
G_{D}(x, y)=G_{D^{*}}(x, y)+\mathbb{E}^{x} G_{D}\left(\mathbf{B}\left(\tau_{D^{*}}\right), y\right) .
$$

Note that $d_{\mathbb{H}^{n}}\left(\mathbf{B}\left(\tau_{D^{*}}\right), e_{n}\right) \geq r_{0} / 4 \geq d_{\mathbb{H}^{n}}(x, y)$ if $\mathbf{B}\left(\tau_{D^{*}}\right) \in \partial D^{*} \backslash \partial D$ and $d_{\mathbb{H}^{n}}\left(e_{n}, y\right) \leq$ $r_{0} / 8$. Hence,

$$
d_{\mathbb{H}^{n}}\left(\mathbf{B}\left(\tau_{D^{*}}\right), y\right) \geq d_{\mathbb{H}^{n}}\left(\mathbf{B}\left(\tau_{D^{*}}\right), e_{n}\right)-d_{\mathbb{H}^{n}}\left(e_{n}, y\right) \geq r_{0} / 8 \geq d_{\mathbb{H}^{n}}(x, y) .
$$


Applying (21) we get, by monotonicity of $U$ with respect to the hyperbolic distance,

$$
G_{D}\left(\mathbf{B}\left(\tau_{D^{*}}\right), y\right) \leq C U\left(\mathbf{B}\left(\tau_{D^{*}}\right), y\right) \frac{\delta_{D}(y)}{r_{0}} \leq C U(x, y) \frac{\delta_{D}(y)}{r_{0}} .
$$

It follows that

$$
\begin{aligned}
\mathbb{E}^{x} G_{D}\left(\mathbf{B}\left(\tau_{D^{*}}\right), y\right) & \leq C U(x, y) \frac{\delta_{D}(y)}{r_{0}} \mathbb{P}^{x}\left(\mathbf{B}\left(\tau_{D^{*}}\right) \in \partial D^{*} \backslash \partial D\right) \\
& \leq C(n) U(x, y) \frac{\delta_{D}(x) \delta_{D}(y)}{r_{0}^{2}},
\end{aligned}
$$

which yields

$$
\mathbb{E}^{x} G_{D}\left(\mathbf{B}\left(\tau_{D^{*}}\right), y\right) \leq C(n) U(x, y)\left(1 \wedge \frac{\left(r_{0} \wedge \delta_{D}(x)\right)\left(r_{0} \wedge \delta_{D}(y)\right)}{r_{0}^{2} \wedge d_{\mathbb{H} \mathbb{H}^{n}}(x, y)^{2}}\right) .
$$

Next, by Lemma 5.6,

$$
G_{D^{*}}(x, y) \approx\left(1 \wedge \frac{\delta_{D}(x) \delta_{D}(y)}{d_{\mathbb{H}^{n}}(x, y)^{2}}\right) U(x, y)
$$

with the comparability constant dependent only on $n$. Since

$$
\left(1 \wedge \frac{\delta_{D}(x) \delta_{D}(y)}{d_{\mathbb{H}^{n}}(x, y)^{2}}\right) \leq 2 \frac{\left(r_{0} \wedge \delta_{D}(x)\right)\left(r_{0} \wedge \delta_{D}(y)\right)}{r_{0}^{2} \wedge d_{\mathbb{H}^{n}}(x, y)^{2}},
$$

we have the desired bound in this case, so the proof is complete.

Remark 1 A natural question arises whether the lower bound is of the same magnitude as the upper one. The answer is negative. To see this we take a strip $D=\left\{1<z_{n}<2\right\}$. It has a hyperbolic characteristic equal to $\frac{1}{2} \log 2$. Then from Corollary 4.2 we have

$$
G_{D}(x, y)=\frac{1}{h(x) h(y)} n_{D}(x, y) G_{D}^{\Delta}(x, y) \approx G_{D}^{\Delta}(x, y) .
$$

We know that (see e.g. (30) in [21] for even dimensions)

$$
G_{D}^{\Delta}(x, y) \approx\left(1 \wedge \frac{\delta_{D}(x) \delta_{D}(y)}{|x-y|^{2}}\right) \frac{\left(1+|x-y|^{(n+2) / 2}\right)}{|x-y|^{n-2}} e^{-|x-y| / \pi} .
$$

and this can not be estimated from below as desired.

Corollary 5.8 Let $0<r_{0} \leq 1$ and let $D$ be a $C^{1,1}$ bounded set with the hyperbolic characteristic $r_{0}$. Then we have the following bound of the hyperbolic Poisson kernel of $D$,

$$
P_{D}(x, z) \leq C \frac{1}{z_{n}^{n-1}} \frac{r_{0} \wedge \delta_{D}(x)}{\left(r_{0} \wedge d_{\mathbb{H}^{n}}(x, z)\right)^{2}} U(x, z),
$$

with $C=C(n)$.

Proof By Corollary 4.2 we have $P_{D}(x, z)=h^{2}(z) \frac{\partial G_{D}(x, \cdot)}{\partial n_{z}}$, where $n_{z}$ is the inward unit vector normal to $\partial D$ at $z$ and the directional derivative is computed at $z$. By the general theory for $C^{1,1}$ domains, the Green function $G_{D}(x, z)=0$ for $x \in D, z \in \partial D$. Using Theorem 5.7, we get for $t>0$ small enough

$$
\begin{aligned}
G_{D}\left(x, z+t n_{z}\right)-G(x, z) & =G_{D}\left(x, z+t n_{z}\right) \\
& \leq C \frac{r_{0} \wedge \delta_{D}(x)}{r_{0}^{2} \wedge d_{\mathbb{H}^{n}}\left(x, z+t n_{z}\right)^{2}} U\left(x, z+t n_{z}\right) \delta_{D}\left(z+t n_{z}\right),
\end{aligned}
$$


with $C=C(n)$. Hence by continuity of $U(\cdot, \cdot)$ and of the hyperbolic metric

$$
\frac{\partial G_{D}(x, \cdot)}{\partial n_{z}} \leq C \frac{r_{0} \wedge \delta_{D}(x)}{r_{0}^{2} \wedge d_{\mathbb{H}^{n}}(x, z)^{2}} U(x, z) \lim _{t \rightarrow 0^{+}} \frac{\delta_{D}\left(z+t n_{z}\right)}{t}=C \frac{r_{0} \wedge \delta_{D}(x)}{r_{0}^{2} \wedge d_{\mathbb{H}^{n}}(x, z)^{2}} U(x, z) \frac{1}{z_{n}},
$$

since, by (4), $\lim _{t \rightarrow 0^{+}} \frac{\delta_{D}\left(z+t n_{z}\right)}{t}=\lim _{t \rightarrow 0^{+}} \frac{d_{\mathbb{H}^{n}}\left(z+t n_{z}, z\right)}{t}=1 / z_{n}$. Finally, because $\left(1 / z_{n}\right) h^{2}(z)=z_{n}^{1-n}$, we get the desired estimate.

\section{Sharp Green Function Estimates for a Hyperbolic Ball}

This section is devoted to finding sharp two sided estimates of the hyperbolic Green function of a ball. The following theorem, which will be proved by a series of lemmas, is the main result of the paper.

Theorem 6.1 Let $B=B\left(x_{0}, R\right)$ be a hyperbolic ball of any center $x_{0}$ and any radius $R>0$. Then for all $x, y \in B$,

$$
G_{B}(x, y) \approx\left(1 \wedge \frac{\left(1 \wedge \delta_{B}(x)\right)\left(1 \wedge \delta_{B}(y)\right)}{1 \wedge d_{\mathbb{H}^{n}}(x, y)^{2}}\right) U(x, y) .
$$

Moreover, for $x \in B$ and $z \in \partial B$,

$$
P_{B}(x, z) \approx \frac{1}{z_{n}^{n-1}} \frac{1 \wedge \delta_{B}(x)}{\left(1 \wedge d_{\mathbb{H}^{n}}(x, z)\right)^{2}} U(x, z) .
$$

The comparability constants depend only on $n$.

We start with the following technical lemma.

Lemma 6.2 Let $M>16$ and $R$ be such that $R \geq \log \left(M^{2}\right)=C_{M}$. Assume for $x, y \in B_{R}$ that $\delta_{B}(x), \delta_{B}(y)>\log \left(M^{2}\right), d_{\mathbb{H}^{n}}(x, y) \geq 1$ and $x_{n}=y_{n}$. Then for $z \in \partial B_{R}$ we have

$$
\frac{z_{n}}{(|x-z||y-z|)^{2}} \leq \frac{24}{M} \frac{1}{|x-y|^{2}} \max \left\{\frac{x_{n}}{|x-z|^{2}}, \frac{y_{n}}{|y-z|^{2}}\right\} \text {. }
$$

Proof First, let us observe that assumption $\delta_{B}(x)>C_{M}$ is equivalent to $x \in B_{R-C_{M}}$, and hence (see Eq. 18),

$$
e^{-R} M^{2}=e^{-\left(R-C_{M}\right)}<x_{n}<e^{\left(R-C_{M}\right)}=e^{R} M^{-2} .
$$

Next, recall that $\tilde{x}=\left(x_{1}, \ldots, x_{n-1}\right)$. We may assume, without loss of generality, that $|\tilde{x}| \geq|\tilde{y}|$, which, together with $x_{n}=y_{n}$, imply that $|x-y| \leq 2|\tilde{x}|$. By (18), we have for $\delta_{B}(x)>C_{M}$,

$$
\begin{gathered}
|\tilde{x}|^{2}<\sinh ^{2}\left(R-C_{M}\right)-\left(\cosh \left(R-C_{M}\right)-x_{n}\right)^{2} \\
=-1+2 x_{n} \cosh \left(R-C_{M}\right)-x_{n}^{2} \\
<2 x_{n} \cosh \left(R-C_{M}\right)<2 x_{n} e^{R-\log \left(M^{2}\right)} \\
<2 \frac{x_{n} e^{R}}{M^{2}} .
\end{gathered}
$$

Now we pass to the main part of the proof. By the triangle inequality we get

$$
\frac{1}{|x-z||y-z|} \leq 2 \frac{1}{|x-y|} \max \left\{\frac{1}{|x-z|}, \frac{1}{|y-z|}\right\} \text {. }
$$


Hence, the assertion is clear for $z_{n} \leq \frac{x_{n}}{M}$.

For $z \in \partial B_{R}$ such that $x_{n} / M<z_{n}<\cosh R$ we have, by (22), $z_{n}>x_{n} / M>M e^{-R}$ and consequently

$$
\begin{aligned}
|\tilde{z}|^{2} & =-1+2 z_{n} \cosh (R)-z_{n}^{2} \\
> & -1+z_{n} \cosh (R)>\frac{1}{4} z_{n} e^{R} .
\end{aligned}
$$

The above inequality together with (23) imply that

$$
|\tilde{x}|^{2}<\frac{8 x_{n}|\tilde{z}|^{2}}{M^{2} z_{n}}<\frac{8|\tilde{z}|^{2}}{M} .
$$

In particular, we have $|z-x|^{2}>\frac{1}{12}|\tilde{z}|^{2}$. Recalling that $|x-y|^{2} \leq 4|\tilde{x}|^{2}$ and $x_{n}=y_{n}$, we obtain for $x_{n} / M<z_{n}<\cosh R$,

$$
\begin{aligned}
\frac{z_{n}}{|x-z|^{2}}= & \frac{y_{n}}{|x-y|^{2}} \frac{z_{n}}{y_{n}} \frac{|x-y|^{2}}{|x-z|^{2}}<48 \frac{y_{n}}{|x-y|^{2}} \frac{z_{n}}{y_{n}} \frac{|\tilde{x}|^{2}}{|\tilde{z}|^{2}} \\
& <384 \frac{y_{n}}{|x-y|^{2}} \frac{z_{n}}{y_{n}} \frac{x_{n}}{M^{2} z_{n}}<\frac{24}{M} \frac{y_{n}}{|x-y|^{2}} .
\end{aligned}
$$

This follows

$$
\frac{z_{n}}{(|x-z||y-z|)^{2}} \leq \frac{24}{M} \frac{1}{|x-y|^{2}} \frac{y_{n}}{|y-z|^{2}},
$$

which implies the conclusion.

It remains to consider $z_{n} \geq \cosh R$. In this case, by (22),

$$
|x-z| \geq z_{n}-x_{n} \geq \cosh R-e^{R} M^{-2} \geq \frac{1}{3} e^{R},
$$

hence

$$
\frac{z_{n}}{|x-z|^{2}} \leq 9 e^{-R}
$$

Furthermore, Eq. 23 gives us

$$
e^{-R} \leqslant \frac{2}{M^{2}} \frac{x_{n}}{\max \left\{|\tilde{x}|^{2},|\tilde{y}|^{2}\right\}} \leqslant \frac{8}{M^{2}} \frac{x_{n}}{|x-y|^{2}} .
$$

The last two inequalities complete the proof.

The lemma below is a crucial step in proving the lower bound of the Green function of a ball. Let us mention that the proof of the lower bound is much more difficult than the proof of the upper bound.

Lemma 6.3 Let $B$ be any hyperbolic ball with a radius $R$. There exists a constant $C^{*}=$ $C^{*}(n) \geq 2$ such that for $x, y \in B$ with $d_{\mathbb{H}^{n}}(x, y) \geq 1$ and $\delta_{B}(x), \delta_{B}(y) \geq C^{*}$ we have

$$
G_{B}(x, y) \geq \frac{1}{2} U(x, y) .
$$

Furthermore, without the assumption $d_{\mathbb{H}^{n}}(x, y) \geq 1$ we have

$$
G_{B}(x, y) \geq \tilde{C} U(x, y) .
$$

for some $\tilde{C}=\tilde{C}(n) \geq 0$. 
Proof We may choose the ball $B=B_{R}$. Since the function $G_{B}$ is invariant with respect to isometries of $\mathbb{H}^{n}$ which map $B$ onto $B$ we may assume that $x_{n}=y_{n} \leqslant 1$ which follows from Proposition 2.2. Relaying on the formula

$$
G_{B}(x, y)=U(x, y)-\mathbb{E}^{x} U\left(\mathbf{B}\left(\tau_{B}\right), y\right), x, y \in B,
$$

our aim is to show that for $\delta_{B}(x), \delta_{B}(y)$ sufficiently large the term $\mathbb{E}^{x} U\left(\mathbf{B}\left(\tau_{B}\right), y\right)$ is smaller than $\frac{1}{2} U(x, y)$. Next,

$$
\mathbb{E}^{x} U\left(\mathbf{B}\left(\tau_{B}\right), y\right)=\int_{\partial B} U(z, y) P_{B}(x, z) d z,
$$

where $P_{B}(x, z)$ stands for the Poisson kernel of the ball $B$ with respect to the Euclidean surface measure on $\partial B$. Assume that $\delta_{B}(x) \geq 1$. Applying Corollary 5.8 with $r_{0}=1$ and noting that $d_{\mathbb{H}^{n}}(x, z) \geq \delta_{B}(x) \geq 1, z \in \partial B$, we have

$$
P_{B}(x, z) \leqslant C \frac{1}{z_{n}^{n-1}} \frac{1 \wedge \delta_{B}(x)}{\left(1 \wedge d_{\mathbb{H}^{n}}(x, z)\right)^{2}} U(x, z)=C \frac{1}{z_{n}^{n-1}} U(x, z),
$$

with $C=C(n)$. Then, we may write

$$
\mathbb{E}^{x} U\left(\mathbf{B}\left(\tau_{B}\right), y\right) \leqslant C \int_{\partial B} U(x, z) U(z, y) \frac{1}{z_{n}^{n-1}} d z .
$$

Furthermore, for $d_{\mathbb{H}^{n}}(u, w) \geq 1$ we have (see (17))

$$
U(u, w) \approx \frac{1}{\left(\cosh d_{\mathbb{H}^{n}}(u, w)\right)^{n-1}} \approx\left(\frac{u_{n} w_{n}}{|u-w|^{2}}\right)^{n-1} .
$$

Let $M \geq 16$. Applying the last estimate together with Lemma 6.2, for $\delta_{B}(x), \delta_{B}(y)>$ $\log \left(M^{2}\right)$, we get

$$
U(x, z) U(z, y) \frac{1}{z_{n}^{n-1}} \leq C \frac{1}{M^{n-1}} U(x, y)\left[\left(\frac{x_{n}}{|x-z|^{2}}\right)^{n-1}+\left(\frac{y_{n}}{|y-z|^{2}}\right)^{n-1}\right], z \in \partial B,
$$

where we used the assumption $x_{n}, y_{n} \leqslant 1$. The above estimates imply that

$$
\begin{gathered}
\int_{\partial B} U(x, z) U(z, y) \frac{1}{z_{n}^{n-1}} d z \\
\leq C \frac{1}{M^{n-1}} U(x, y) \int_{\partial B}\left[\left(\frac{x_{n}}{|x-z|^{2}}\right)^{n-1}+\left(\frac{y_{n}}{|y-z|^{2}}\right)^{n-1}\right] d z .
\end{gathered}
$$

By Corollary A.1, the last integral is bounded by a constant dependent only on $n$. Hence,

$$
\int_{\partial B} U(x, z) U(z, y) \frac{1}{z_{n}^{n-1}} d z \leq C \frac{1}{M^{n-1}} U(x, y) \leq \frac{1}{2} U(x, y)
$$

for $M$ sufficiently large, which gives the first inequality from the assertion. Next, consider $d_{\mathbb{H}^{n}}(x, y)<1$. Since $\delta_{D}(x) \geq C^{*} \geq 2$, then $B(x, 2) \subset B$ and by Lemma 5.6,

$$
G_{B}(x, y) \geq G_{B(x, 2)}(x, y) \approx U(x, y) .
$$

This ends the proof.

Lemma 6.4 Let $C^{*}$ be a constant found in Lemma 6.3. Then for any $R \geq 4 C^{*}$ and $x, y \in$ $B_{R}$ with $d_{\mathbb{H}^{n}}(x, y) \geq 4 C^{*}+1$ we have

$$
G_{B_{R}}(x, y) \geq C\left(1 \wedge \delta_{B_{R}}(x)\right)\left(1 \wedge \delta_{B_{R}}(y)\right) U(x, y),
$$

where $C=C(n)$. 
Proof Let $B=B_{R}$ and $D=B \cap B\left(x_{0}, 2 C^{*}\right)$, where $x_{0}=\left(0, \ldots, 0, e^{-R}\right) \in \partial B$. We may assume that $d_{\mathbb{H}^{n}}\left(x, x_{0}\right)=\delta_{B}(x)$. Let $S=\left\{z: d_{\mathbb{H}^{n}}\left(z, x_{0}\right)=2 C^{*}, \delta_{B}(z) \geq C^{*}\right\}$. By Lemma 6.3, it is enough to consider the case (A): $\delta_{B}(x) \leq C^{*} \leq \delta_{B}(y)$ and the case (B): $\delta_{B}(x) \leq \delta_{B}(y) \leq C^{*}$.

Case (A): We relax the assumption on $d_{\mathbb{H}^{n}}(x, y)$ to $d_{\mathbb{H}^{n}}(x, y)>2 C^{*}+1$. Since $\delta_{B}(x) \leq$ $C^{*}$, then, by Lemma 5.4, $\mathbb{P}^{x}\left(\mathbf{B}\left(\tau_{D}\right) \in S\right) \geq C(n) \delta_{B}(x)$. For $z \in S$ observe that $d_{\mathbb{H}^{n}}(y, z) \geq$ $d_{\mathbb{H}^{n}}(x, y)-d_{\mathbb{H}^{n}}(x, z) \geq 2 C^{*}+1-2 C^{*}=1$. Applying Lemma 6.3 we have $G_{B}(z, y) \geq$ $(1 / 2) U(z, y)$. Moreover $1 \leq d_{\mathbb{H}^{n}}(y, z) \leq d_{\mathbb{H}^{n}}(x, y)+d_{\mathbb{H}^{n}}(x, z) \leq d_{\mathbb{H}^{n}}(x, y)+2 C^{*}$. Hence, by (17) we have $U(z, y) \geq c U(x, y)$ with $c=c(n)$. This yields

$$
\begin{aligned}
G_{B}(x, y) & =\mathbb{E}^{x} G_{B}\left(\mathbf{B}\left(\tau_{D}\right), y\right) \\
& \geq \inf _{z \in S} G_{B}(z, y) \mathbb{P}^{x}\left(\mathbf{B}\left(\tau_{D}\right) \in S\right) \\
& \geq c U(x, y) \delta_{B}(x),
\end{aligned}
$$

which completes the proof in the first case.

Case (B): Let $d_{\mathbb{H}^{n}}(x, y)>4 C^{*}+1$. Observe that for $z \in S$ we have $d_{\mathbb{H}^{n}}(y, z) \geq$ $d_{\mathbb{H}^{n}}(x, y)-d_{\mathbb{H}^{n}}(x, z) \geq 2 C^{*}+1$ so, by case (A), we have

$$
G_{B}(z, y) \geq C U(z, y) \delta_{B}(y) \geq C U(x, y) \delta_{B}(y) .
$$

Therefore

$$
\begin{aligned}
G_{B}(x, y) & =\mathbb{E}^{x} G_{B}\left(\mathbf{B}\left(\tau_{D}\right), y\right) \\
& \geq \inf _{z \in S} G_{B}(z, y) \mathbb{P}^{x}\left(\mathbf{B}\left(\tau_{D}\right) \in S\right) \\
& \geq C U(x, y) \delta_{B}(x) \delta_{B}(y),
\end{aligned}
$$

with $C=C(n)$. The proof is completed.

Lemma 6.5 Let $C^{*}$ be a constant found in Lemma 6.3. Then for any $R \geq 20 C^{*}$ and $x, y \in$ $B_{R}$ with $d_{\mathbb{H}^{n}}(x, y)<4 C^{*}+1$ we have

$$
G_{B_{R}}(x, y) \geq C\left(1 \wedge \frac{\delta_{B_{R}}(x) \delta_{B_{R}}(y)}{d_{\mathbb{H}^{n}}(x, y)^{2}}\right) U(x, y),
$$

where $C=C(n)$.

Proof Without loss of generality we may assume that instead of $B_{R}$ we may take the ball $B=B\left(x^{*}, R\right)$, where $x^{*}=\left(0, \ldots, 0, x_{n}^{*}\right)$ and $x=e_{n}$. If $\delta_{B}(x)>2 d_{\mathbb{H}^{n}}(x, y)$, then $B\left(e_{n}, 2 d_{\mathbb{H}^{n}}(x, y)\right) \subset B_{R}$, and consequently $G_{B\left(e_{n}, 2 d_{\mathbb{H}^{n}}(x, y)\right)}(x, y) \leqslant G_{B_{R}}(x, y)$. Next, by Lemma 5.6, $G_{B\left(e_{n}, 2 d_{\mathbb{H}^{n}}(x, y)\right)}(x, y)$ is comparable with $U(x, y)$, which implies the required inequality. If $\delta_{B}(x) \leq 2 d_{\mathbb{H}^{n}}(x, y)$, let us denote by $B^{\prime} \subset B_{R}$ the ball of radius $4 d_{\mathbb{H}^{n}}(x, y)$ tangent to $B$ at the point $x_{0}$ such that $d_{\mathbb{H}^{n}}\left(x, x_{0}\right)=\delta_{B}(x)$ and by $B^{\prime \prime} \subset B$ the ball of radius $4 d_{\mathbb{H}^{n}}(x, y)$ tangent to $B$ at the point $y_{0}$ such that $d_{\mathbb{H}^{n}}\left(y, y_{0}\right)=\delta_{B}(y)$. Such choice of $B^{\prime \prime}$ is possible since $\delta_{B}(y) \leq 3 d_{\mathbb{H}^{n}}(x, y)$. Let $D^{*}$ be the smallest convex set containing $B^{\prime} \cup B^{\prime \prime}$. Then $D^{*} \subset B \cap B\left(e_{n}, 20 C^{*}+5\right)$. It is clear that $D^{*}$ is $C^{1,1}, \delta_{B}(x)=\delta_{D^{*}}(x)$ and $\delta_{B}(y)=\delta_{D^{*}}(y)$. Then by Lemma 5.6,

$$
G_{B}(x, y) \geq G_{D^{*}}(x, y) \geq C\left(1 \wedge \frac{\delta_{B_{R}}(x) \delta_{B_{R}}(y)}{d_{\mathbb{H}^{n}}(x, y)^{2}}\right) U(x, y),
$$

and the conclusion follows. 
Proof of Theorem 6.1 The upper bound for the Green function follows from Theorem 5.7.

Let $R_{0}=20 C^{*}$, where $C^{*}$ is the constant found in Lemma 6.3. The lower bound for the Green function in the case $R \leq R_{0}$ follows from Lemma 5.6. Finally the lower bound in the case $R \geq R_{0}$ follows from Lemma 6.4 and Lemma 6.5.

The upper bound for the Poisson kernel follows from Corollary 5.8, while the lower bound is proved by the same arguments as in Corollary 5.8 but with the reverse of the relevant inequalities.

Acknowledgments G. Serafin was supported by the National Science Centre, Poland, grant no. 2015/18/E/ST1/00239. M. Ryznar and T. Żak were partially supported by the National Science Centre, Poland, grant no. 2015/17/B/ST1/01043. We thank the referee for valuable comments and remarks which improved the presentation of the paper.

Open Access This article is licensed under a Creative Commons Attribution 4.0 International License, which permits use, sharing, adaptation, distribution and reproduction in any medium or format, as long as you give appropriate credit to the original author(s) and the source, provide a link to the Creative Commons licence, and indicate if changes were made. The images or other third party material in this article are included in the article's Creative Commons licence, unless indicated otherwise in a credit line to the material. If material is not included in the article's Creative Commons licence and your intended use is not permitted by statutory regulation or exceeds the permitted use, you will need to obtain permission directly from the copyright holder. To view a copy of this licence, visit http://creativecommonshorg/licenses/by/4.0/.

\section{Appendix}

Proposition A.1 Let $a>\frac{n-1}{2}, n \geqslant 2$, and let $B$ be any Euclidean ball in $\mathbb{R}^{n}$. Then we have

$$
\int_{\partial B} \frac{1}{|x-z|^{2 a}} d z \approx \frac{1}{\left(\rho_{B}(x)\right)^{2 a-n+1}}, x \in B
$$

where the comparability constant depends only on a and $n$, and $\rho_{B}(x)$ stands for Euclidean distance between $x$ and $\partial B$.

Proof Without loss of generality we may assume $B=B(0,1)$ and $x=\left(0, \ldots, 0, x_{n}\right)$, where $x_{n}>0$. It is clear that for $x_{n}<\frac{1}{2}$ the integral is comparable with a constant. For $x_{n} \geqslant \frac{1}{2}$ we rewrite it as follows

$$
\begin{aligned}
\int_{\partial B} \frac{1}{|x-z|^{2 a}} d z= & \int_{\partial B} \frac{1}{\left(z_{1}^{2}+\ldots+z_{n-1}^{2}+\left(x_{n}-z_{n}\right)^{2}\right)^{a}} d z \\
= & \Omega_{n-2} \int_{-1}^{1} \frac{\left(1-r^{2}\right)^{(n-3) / 2}}{\left(1^{2}-r^{2}+\left(x_{n}-r\right)^{2}\right)^{a}} d r \\
& =\Omega_{n-2} \int_{0}^{2} \frac{(u(2-u))^{(n-3) / 2}}{\left(\left(1-x_{n}\right)^{2}+2 x_{n} u\right)^{a}} d u \\
& \approx \int_{0}^{1} \frac{u^{(n-3) / 2}}{\left(\left(1-x_{n}\right)^{2}+2 x_{n} u\right)^{a}} d u
\end{aligned}
$$

where $\Omega_{n-2}$ is the surface area of the unit sphere in $\mathbb{R}^{n-1}$. Next, we substitute $u=\frac{\left(1-x_{n}\right)^{2}}{2 x_{n}} s$ and get

$$
\left(\frac{2 x_{n}}{\left(1-x_{n}\right)^{2}}\right)^{a-(n-1) / 2} \int_{0}^{2 x_{n} /\left(1-x_{n}\right)^{2}} \frac{s^{(n-3) / 2}}{(1+s)^{a}} d s .
$$

Since for $x_{n} \geqslant \frac{1}{2}$ it holds $x_{n} \approx 1$ and $4 x_{n} /\left(1-x_{n}\right)^{2}>8$, we obtain the required estimate. 
Corollary A.1 Let $\gamma>0$ and let $B_{R}, R>1$, be defined as in (18). If $x \in B_{R}$ and $\delta_{B_{R}}(x)>1$, then

$$
\int_{\partial B_{R}} \frac{x_{n}^{2 \gamma}}{|x-z|^{2 \gamma+n-1}} d z \approx 1,
$$

where the comparability constant depends only on $\gamma$ and $n$.

Proof We fix $R>1$ and denote $B=B_{R}$. In view of the previous proposition, it is enough to show that $\rho_{B}(x) \approx x_{n}$ whenever $\delta_{B}(x)>1$. The inequality $\rho_{B}(x) \leq x_{n}$ is clear. On the other hand, we may write

$$
\rho_{B}(x)=\sinh R-\sqrt{|\tilde{x}|^{2}+\left(x_{n}-\cosh R\right)^{2}} \approx \frac{\sinh ^{2} R-|\tilde{x}|^{2}-\left(x_{n}-\cosh R\right)^{2}}{\sinh R} .
$$

The assumption $\delta_{B}(x)>1$ implies $x \in B_{R-1}$, which gives us

$$
\begin{gathered}
\sinh ^{2} R-|\tilde{x}|^{2}-\left(x_{n}-\cosh R\right)^{2} \\
>\sinh ^{2} R-\sinh ^{2}(R-1)+\left(x_{n}-\cosh (R-1)\right)^{2}-\left(x_{n}-\cosh R\right)^{2} \\
=2 x_{n}(\cosh R-\cosh (R-1)) \approx x_{n} \cosh R .
\end{gathered}
$$

Finally, we get

which ends the proof.

$$
\rho_{B}(x) \geq C(n) x_{n} \frac{\cosh R}{\sinh R} \approx x_{n},
$$

\section{References}

1. Blumenthal, R.M., Getoor, R.K.: Markov Processes and Potential Theory. Pure and Applied Mathematics, vol. 29. Academic Press, New York (1968)

2. Bogdan, K.: Sharp estimates for the Green function in Lipschitz domains. J. Math. Anal. Appl. 243, 326-337 (2000)

3. Bogdan, K., Jakubowski, T.: Estimates of the Green function for the fractional Laplacian perturbed by gradient. Pot. Anal. 36, 455-481 (2012)

4. Bogus, K., Byczkowski, T., Małecki, J.: Sharp estimates of green function of hyperbolic Brownian motion. Studia Math. 228(3), 197-222 (2015)

5. Byczkowski, T., Małecki, J.: Poisson kernel and Green function of the ball in real hyperbolic spaces. Potential Anal. 27, 1-16 (2007)

6. Byczkowski, T., Graczyk, P., Stós, A.: Poisson kernels of half-spaces in real hyperbolic spaces. Revista Math. Iberoamericana 23(1), 85-126 (2007)

7. Byczkowski, T., Ryznar, M.: Hitting distribution of geometric Brownian motion. Studia Math. 173(1), 19-38 (2006)

8. Cammarota, V., Orsingher, E.: Hitting spheres on hyperbolic spaces. Teor. Veroyatn. Primen. 57, 560587 (2013)

9. Chung, K.L., Zhao, Z.: From Brownian Motion to Schrödinger's Equation. Springer (1995)

10. Chung, K.L., Walsh, J.B.: Markov Processes, Brownian Motion, and Time Symmetry, 2nd edn. Grundlehren der Mathematischen Wissenschaften, vol. 249. Springer (2005)

11. Cranston, M., Fabes, E., Zhao, Z.: Conditional gauge and potential theory for the Schrödinger operator. Trans. AMS 307(1), 171-194 (1988)

12. Davies, E.B.: Heat Kernels and Spectral Theory. Cambridge Univ. Press (1989)

13. Ethier, S., Kurtz, T.G.: Markov Processes. Characterization and Convergence. Wiley, New York (1986)

14. Helgason, S.: Groups and Geometric Analysis. Academic Press (1984)

15. Hueber, H., Sieveking, M.: Uniform bounds for quotients of Green functions on $C^{1,1}$ domains. Ann. Inst. Fourier 32(1), 105-117 (1982)

16. Hunt, G.A.: Markov processes and potentials I, II and III. Illinois J. Math. 1, 44-93 and 316-369 (1957). and Illinois J. of Math. 2, 151-213, (1958) 
17. Matsumoto, H.: Closed form formulae for the heat kernels and the Green functions for the Laplacians on the symmetric spaces of rank one. Bull. Sc. Math. 125(6-7), 553-581 (2001)

18. Ratcliffe, J.G.: Foundations of Hyperbolic Manifolds, Graduate Texts in Math, vol. 149. Springer (2006)

19. Revuz, D., Yor, M.: Continuous Martingales and Brownian Motion, 3rd edn. Springer (2005)

20. Ryznar, M., Żak, T.: Exit time of a hyperbolic $\alpha$-stable process from a halfspace or a ball. Potential Anal. 45(1), 83-107 (2016)

21. Serafin, G.: Potential theory of hyperbolic Brownian motion in tube domains. Colloq. Math. 135, 27-52 (2014)

22. Serafin, G.: On potential theory of hyperbolic Brownian motion with drift. Probab. Math. Statist. 40(1), $1-22(2020)$

23. Symeonidis, E.: The Poisson integral for a ball in spaces of constant curvature. Commentationes Math. Univer. Carolinae 44(3), 437-460 (2003)

24. Zhao, Z.: Green function for Schrödinger operator and conditioned Feynman-Kac gauge. J. Math. Anal. Appl. 116, 309-334 (1986)

Publisher's Note Springer Nature remains neutral with regard to jurisdictional claims in published maps and institutional affiliations. 